\title{
Elliptic and Parabolic Singular Perturbations in the Kinetic Theory of Enzymes (*).
}

\author{
L. S. Frank - W. D. WendT
}

Summary. - A family of semilinear parabolic operators $\mathfrak{A}_{\varepsilon}$ and corresponding elliptic operators $\mathfrak{U}_{\varepsilon, \infty}$ is considered, $\varepsilon$ being a small parameter and the reduced problem being characterized by the presence of a free boundary. This kind of operators appears in the kinetic theory of artificial membranes with enzymotic activity.

\section{Introduction.}

In the Kinetie theory of membranes with enzymotic activity, a second order semilinear parabolic operator appears to be a suitable mathematical model for describing the dynamical process for the corresponding concentrations. The nonlinearity of this operator is affected by the presence of two positive parameters: $\varepsilon$, the so-called Michaelis' constant, and $\lambda$, the latter being connected with the ratio of initial concentrations of the enzyme and the substratum. For several realistic membranes, $\varepsilon$ is small compared to $\lambda$ and the data of the problem ([12]).

For each $\varepsilon>0$ fixed, the mathematical model above fits the classical framework of Fréchet differentiable nonlinear operators. One can view this model as a family of perturbations (regular or singular, according to the magnitude of $\lambda$ ) of some reduced parabolic operator with a piecewise constant discontinuous nonlinearity. The same is also true for the corresponding elliptic stationary problem.

Further, for the stationary problem, there exists a critical value $\lambda_{c}$ of the second parameter $\lambda$, such that for $\lambda<\lambda_{c}$ the original problem is a regular perturbation of the "reduced" one, whereas for $\lambda>\lambda_{e}$, it becomes a singular perturbation and is characterized by the presence of boundary layers located in a neighbourhood of the free boundary of the solution to the "reduced" problem. The set $E_{c}$ of zeros of the corresponding critical solution $u^{\lambda_{c}}$ plays an important role in the investigation of the reduced problem.

One of the central results presented here is the sharp error estimate in the $H_{1}$-norm for the difference of the solutions to the perturbed and reduced problems. Further, $\lambda_{c}$ is investigated as a functional of the data of the reduced problem.

$\left.{ }^{*}\right)$ Entrata in Redazione il 2 ottobre 1984.

Indirizzo degli AA.: L. S. FRANK: Department of Mathematics, University of Nijmegen, Toernooiveld, 6525 ED Nijmegen, The Netherlands; W. D. WendT: Department of Applied Mathematics, University of Bonn, Wegelerstr. 10, D-53 Bonn 1, West Germany. 
Now the contents of the paper will be briefly sketched.

Part I deals with the stationary problem. Section 1.1 contains existence, uniqueness and regularity results as well as continuity results for the operators considered. In section 1.2, asymptotic solutions (for $\varepsilon \downarrow 0$ ) of the perturbed stationary problem are constructed under some regularity assumptions on the free boundary and the sharp $H_{1}$-estimate for the difference $w_{e}$ between the solutions of the reduced and the perturbed problems is established. Namely, the following two-sided estimate holds with some constant $C: C^{-1} \varepsilon^{3 / 4} \leqslant\left\|w_{\varepsilon}\right\|_{H_{1}} \leqslant C \varepsilon^{3 / 4}$. The special case of a piecewise linear nonlinearity plays an important role and allows a considerable simplification in the construction of asymptotic solutions in the general case, as well. A one-sided estimate of the form $\left\|w_{\varepsilon}\right\|_{B_{1}} \leqslant C \varepsilon^{1 / 2}$, whose proof was merely based on the monotonicity of the nonlinearity and did not require regularity assumptions on the free boundary, had previously been given in [2], (see also [16]).

In section 1.3, properties of the critical value $\lambda_{c}$ are stated and an improved convergence result of the form $\left\|w_{\varepsilon}\right\|_{H_{1}} \leqslant C \varepsilon\left(\lambda_{c}-\lambda\right)^{-1}$ is established when $\lambda<\lambda_{c}$. Sections 1.4 and 1.5 contain investigation of $\lambda_{c}$ as a functional of the data.

Estimates of $\lambda_{c}$ from above and from below are indicated and the formula for the Fréchet derivative of $\lambda_{c}$ stated in $[6,8]$ is proved. In section 1.6 , the asymptotic behaviour for $\lambda \rightarrow \infty$ of the solution of the reduced problem and of the corresponding free boundary are investigated.

In Part II, the nonstationary problem is considered.

Section 2.1 contains existence, uniqueness and regularity results. In section 2.2 , it is shown that the solution of the stationary problem is asymptotically stable in $H_{1}$ uniformly with respect to $\varepsilon$. In section 2.3 , an estimate for the difference of the solutions to the nonstationary perturbed and reduced problems is proved. Sections 2.4 and 2.5 deal with nonnegative solutions and special solutions of the Cauchy problem for the reduced equation. In sections 2.6 and 2.7 short time asymptotic results and asymptotics for $\lambda \rightarrow \infty$ are presented. Their proof will be given elsewhere.

Several results in this paper have been announced in [6-8].

The list of references contains essentially papers which are (to the best of the authors' knowledge) tightly connected with the topics presented here. We refer to $[15,14,4],[3],[5,11],[1,13,10]$ and $[16,18-20]$ for more information concerning variational inequalities, maximal monotone operators, variational calculus and convex analysis, free boundary problems and singular perturbation theory.

\section{0. - Notation. Statement of the problem.}

Let $U \subset \boldsymbol{R}^{n}$ be a bounded domain with $C^{\infty}$-boundary $\partial U$ and let $\boldsymbol{R}_{+}=(0, \infty)$. Denote,

$$
Q=U \times \boldsymbol{R}_{+}, \quad Q_{T}=U \times(0, T), \quad \Gamma=\partial U \times \boldsymbol{R}_{+}, \quad \Gamma_{T}=\partial U \times[0, T) .
$$


Let the function $f \in C^{\circ}(\boldsymbol{R}, \boldsymbol{R})$ be piecewise continuously differentiable: $f \in C^{1}\left(\left[s_{k}, s_{k_{+1}}\right]\right), \forall k \in\{0, \ldots, r-1\}$, where $-\infty=s_{0}<s_{1}<\ldots<s_{r}=\infty$. It is also assumed that

$$
\left\{\begin{array}{l}
f(0)=0, \\
0 \leqslant f^{\prime}(s) \leqslant L\left(1+s^{2}\right)^{-1}, \quad \forall s \in \boldsymbol{R} \backslash\left\{s_{1}, \ldots, s_{r-1}\right\},
\end{array}\right.
$$

where $L>0$ is a constant.

As a consequence of $(0.2), f(s)$ is monotonically increasing on $\boldsymbol{R}$ and, moreover, there exist the limits

$$
\lim _{s \rightarrow \pm \infty} f(s)=f_{ \pm \infty}, \quad-\infty<f_{-\infty} \leqslant f_{+\infty}<+\infty
$$

He(s) being Heaviside's function, we associate with $f(s)$ the following function

$$
f_{0}(s)=f_{+\infty} \mathfrak{H}(s)+f_{-\infty} \mathfrak{H}(-s), \quad \forall s \in \boldsymbol{R} \backslash\{0\}, f_{0}(0)=0
$$

We also denote by $F(s)$ and $F_{0}(s)$ the primitive functions of $f(s)$ and $f_{0}(s)$ normalized by the condition: $F(0)=0, F_{0}(0)=0$. Let $\left(a_{k j}(x, t)\right)_{1 \leqslant k, j \leqslant n}$ be uniformly with respect to $(x, t) \in \bar{Q}$ positive definite, and let $a_{k j}(x, t) \in C^{\infty}(\bar{Q})$. It is assumed that the family of operators

$$
t \rightarrow A\left(x, t, \frac{\partial}{\partial x}\right)=-\sum_{1 \leqslant k, j \leqslant n} \frac{\partial}{\partial x_{j}} a_{k j}(x, t) \frac{\partial}{\partial x_{k}}, \quad a_{k j} \in C^{\infty}(\bar{Q}),
$$

stabilizes, as $t \rightarrow+\infty$, to the operator:

$$
A_{\infty}\left(x, \frac{\partial}{\partial x}\right)=-\sum_{1 \leqslant k, j \leqslant n} \frac{\partial}{\partial x_{j}} a_{k j}^{\infty}(x) \frac{\partial}{\partial x_{k}}, \quad a_{k i}^{\infty} \in C^{\infty}(\bar{U}) .
$$

The following initial-boundary value problem $\mathfrak{A}_{\varepsilon}^{\lambda}$ is considered:

$$
\begin{cases}\frac{\partial u_{\varepsilon}^{\lambda}}{\partial t}+A\left(x, t, \frac{\partial}{\partial x}\right) u_{\varepsilon}^{\lambda}+\lambda f\left(\frac{u_{\varepsilon}^{\lambda}}{\varepsilon}\right)=g(x, t), & (x, t) \in Q \\ u_{\varepsilon}^{\lambda}(x, 0)=\psi(x), & x \in \bar{U}, \\ \pi_{0} u_{\varepsilon}^{\lambda}\left(x^{\prime}, t\right)=\phi\left(x^{\prime}, t\right), & \left(x^{\prime}, t\right) \in \Gamma\end{cases}
$$

where $\lambda, \varepsilon$ are positive parameters; $\pi_{0}$ is the restriction operator to $\Gamma$, the data are supposed to have the following regularity:

$$
g \in C^{0}(\bar{Q}), \quad \psi \in C^{2}(\mathrm{U}), \quad \phi \in C^{2,1}(\Gamma)
$$


to satisfy the compatibility condition:

$$
\pi_{0} \psi\left(x^{\prime}\right)=\phi\left(x^{\prime}, 0\right), \quad \forall x^{\prime} \in \partial U
$$

and to stabilize to

$$
g_{\infty} \in C^{0}(\bar{U}), \quad \phi_{\infty} \in C^{2}(\partial U)
$$

as $t \rightarrow+\infty$.

Here, as usual, $C^{0}(\bar{Q})$ is the space of all continuous in $\bar{Q}$ real-valued functions, $C^{2}(\bar{U})$ is the space of all twice continuously differentiable real-valued functions in $\bar{U}$ and $C^{2,1}(\Gamma)$ is the space of all continuous real-valued functions on $\Gamma$ such that their first derivatives with respect to $\left(x^{\prime}, t\right) \in T$ and the second derivatives with respect to $x^{\prime} \in \partial U$ are continuous functions. The parameter $\varepsilon$ is assumed to be small compared to $\lambda: 0<\varepsilon \ll \lambda$.

Along with the problem $\mathfrak{A}_{\varepsilon}^{\lambda}$, we also consider the corresponding stationary problem $\mathfrak{A}_{\varepsilon, \infty}^{\lambda}$ :

$$
\begin{cases}A_{\infty}\left(x, \frac{\partial}{\partial x}\right) u_{\varepsilon, \infty}^{\lambda}+\lambda f\left(\frac{u_{\varepsilon, \infty}^{\lambda}}{\varepsilon}\right)=g_{\infty}(x), & x \in U, \\ \pi_{0} u_{\varepsilon, \infty}^{\lambda}\left(x^{\prime}\right)=\phi_{\infty}\left(x^{\prime}\right), & x^{\prime} \in \partial U .\end{cases}
$$

Example 0.1. - With $A=-\Delta, f(s)=s(1+|s|)^{-1}$, the problem (0.6) appears in the kinetic theory of membranes with enzymotic activity ([12]).

In this case $f_{0}(s)=\operatorname{sgn} s, \forall s \in \boldsymbol{R} \backslash\{0\}$. Since in applications one is essentially interested in non-negative solutions ( $u_{\varepsilon}^{\lambda}$ is interpreted as the dynamical concentration of the enzyme in this case), one can also define $f(s)$ to be $f(s)=s_{+}(1+s)^{-1}$ with $s_{+}=\max (s, 0)$. Then one has $f_{0}(s)=\mathscr{H}(s)$.

Example 0.2. - Let $l, a_{+}, a_{-} \in \boldsymbol{R}_{+}$be fixed and let $f_{l}$ denote the piecewise linear function

$$
f_{l}(s)=\mathscr{H}(s) \min \left\{l s, a_{+}\right\}+\mathscr{H}(-s) \max \left\{l s,-a_{-}\right\} .
$$

An asymptotic (for $\varepsilon \downarrow 0$ ) solution of $\mathfrak{A}_{\varepsilon, \infty}^{2}$ with $f=f_{b}$ is constructed in section 1.2 below and used in order to investigate the asymptotic behaviour (for $\varepsilon \downarrow 0$ ) of the solution of $\mathfrak{A}_{\varepsilon, \infty}^{\lambda}$ with general $f$.

For a given function $u \in C^{0}(\bar{Q})$ (or $u \in C^{0}(\bar{U})$ ), denote by $E_{+}(u), E_{-}(u)$ the sets where $u>0, u=0, u<0$, respectively, whereas $\chi_{+}(u), \chi_{0}(u), \chi_{-}(u)$ stand for the characteristic functions of these sets. 
We associate with $\mathfrak{H}_{\varepsilon}^{\lambda}$ the following "reduced " problem $\mathfrak{A}^{\lambda}$ :

$$
\begin{cases}\frac{\partial u^{\lambda}}{\partial t}+A\left(x, t, \frac{\partial}{\partial x}\right) u^{\lambda}+\lambda f_{0}\left(u^{\lambda}\right)=g(x, t)\left[1-\chi_{0}\left(u^{\lambda}\right)\right], & (x, t) \in Q, \\ \lambda f_{-\infty} \leqslant g(x, t) \leqslant \lambda f_{+\infty}, & (x, t) \in \operatorname{int} E_{0}\left(u^{\lambda}\right), \\ u^{\lambda}(x, 0)=\psi(x), & x \in \vec{U}, \\ \pi_{0} u^{\lambda}\left(x^{\prime}, t\right)=\phi\left(x^{\prime}, t\right), & \left(x^{\prime}, t\right) \in \Gamma .\end{cases}
$$

The solution $u^{\lambda}$ of $(0.9)$ is supposed to be continuous in $\bar{Q}$, the differential equation and the condition $\lambda f_{-\infty} \leqslant g(x, t) \leqslant \lambda f_{\infty}$ in $(0.9)$ are interpreted in the sense of Schwartz's distributions.

The corresponding stationary «reduced" problem $\mathfrak{H}_{\infty}^{\lambda}$ is stated as follows:

$$
\begin{cases}A_{\infty}\left(x, \frac{\partial}{\partial x}\right) u_{\infty}^{\lambda}+\lambda f_{0}\left(u_{\infty}^{\lambda}\right)=g_{\infty}(x)\left[1-\chi_{0}\left(u_{\infty}^{\lambda}\right)\right], & x \in U, \\ \lambda f_{-\infty} \leqslant g_{\infty}(x) \leqslant \lambda f_{+\infty}, & x \in \operatorname{int} E_{0}\left(u_{\infty}^{\lambda}\right), \\ \pi_{0} u_{\infty}^{\lambda}\left(x^{\prime}\right)=\phi_{\infty}\left(x^{\prime}\right), & x^{\prime} \in \partial U,\end{cases}
$$

where again $u_{\infty}^{\lambda} \in C^{0}(\widetilde{U})$, the differential equation and the condition $\lambda f_{-\infty} \leqslant g_{\infty}(x) \leqslant$ $\leqslant \lambda f_{\infty}$ in $(0.10)$ are interpreted in the distributional sense. The reduced problems $(0.9),(0.10)$ can be reformulated in terms of maximal monotone operators (see [3]).

\section{1. - Stationary problem.}

\subsection{General properties of the operators considered.}

Both $\mathfrak{A}_{\varepsilon, \infty}^{\lambda}$ and $\mathfrak{A}_{\infty}^{\lambda}$ can be equivalently reformulated as variational minimization problems (see, for instance, [5]), where the corresponding functionals

$$
\begin{aligned}
& D_{\varepsilon}^{\lambda}(u)=\int_{U}\left[\frac{1}{2} \sum_{1 \leqslant k, j \leqslant n} a_{k j}^{\infty}(x) \frac{\partial u}{\partial x_{k}} \frac{\partial u}{\partial x_{j}}+\lambda \varepsilon F\left(\frac{u}{\varepsilon}\right)-g_{\infty} u\right] d x, \\
& D^{\lambda}(u)=\int_{U}\left[\frac{1}{2} \sum_{1 \leqslant k, j \leqslant n} a_{k j}^{\infty}(x) \frac{\partial u}{\partial x_{k}} \frac{\partial u}{\partial x_{j}}+\lambda F_{0}(u)-g_{\infty} u\right] d x,
\end{aligned}
$$

are lower semi-continuous, coercive and strictly convex on the hyperplane

$$
\Pi_{\Phi_{\infty}}=\left\{u \in H_{1}(U): u-\Phi_{\infty} \in \stackrel{\circ}{H}_{1}(U)\right\} .
$$

Here, as usual, $H_{1}(U)$ is the Sobolev space of order 1 and $\stackrel{\circ}{H}_{1}(U)$ is the subspace 
of those functions in $H_{1}(U)$, for which traces on $\partial U$ vanish; further $\Phi_{\infty}$ is the solution of the following boundary value problem:

$$
\begin{cases}A_{\infty}\left(x, \frac{\partial}{\partial x}\right) \Phi_{\infty}=g_{\infty}(x), & x \in U, \\ \pi_{0} \Phi_{\infty}\left(x^{\prime}\right)=\phi_{\infty}\left(x^{\prime}\right), & x^{\prime} \in \partial U .\end{cases}
$$

Using the equivalent variational reformulation of $\mathfrak{H}_{\varepsilon, \infty}^{\lambda}, \mathfrak{H}_{\infty}^{\lambda}$ and the classical a priori estimates for linear second order elliptic operators, one gets the following result:

THEOREM 1.1.1. - There exist well-defined solutions

$$
u_{\varepsilon, \infty}^{\lambda} \in C^{1, \alpha}(\bar{U}), \quad u_{\infty}^{\lambda} \in C^{1, \alpha}(\bar{U}), \quad \forall \alpha \in[0,1)
$$

of the problems $\mathfrak{A}_{\varepsilon, \infty}^{\lambda}$ and $\mathfrak{A}_{\infty}^{\lambda}$, respectively.

We use the same notation $\mathfrak{I}_{\varepsilon, \infty}^{\lambda}$ for the operator

$$
\mathfrak{H}_{\varepsilon, \infty}^{\lambda}: H_{2}(U) \rightarrow L_{2}(U) \times H_{3 / 2}(\partial U)
$$

associated with the boundary value problem $(0.8)$, where, as usual, $H_{s}(U)$ and $H_{r}(\partial U)$ stand for the Sobolev spaces (of orders $s$ and. $r$, respectively) of functions in $U$ and on $\partial U$. We are going to state several results concerning the continuity properties of the nonlinear operator $\mathfrak{H}_{\delta, \infty}^{\lambda}$ and its inverse.

Proposition 1.1.2. - For any given $\varepsilon>0$, the mapping (1.1.2) is a Lipsehitzcontinuous homeomorphism.

Propositron 1.1.3. - For any given $\phi_{\infty} \in H_{1 / 2}(\partial U)$, the operator

$$
\left(\mathfrak{Q}_{\varepsilon, \infty}^{\lambda}\right)^{-1}: H_{-1}(U) \rightarrow \Pi_{\Phi_{\infty}}
$$

is Lipschitz-continuous uniformly with respect to $\varepsilon$.

Proposirion 1.1.4. - With $u_{\varepsilon, \infty}^{\lambda}$ the solution of $\mathfrak{H}_{\varepsilon, \infty}^{\lambda}$, the mapping

$$
\overline{\boldsymbol{R}}_{+} \ni \lambda \rightarrow u_{\varepsilon, \infty}^{\lambda} \in H_{\mathbf{1}}(U)
$$

is Lipschitz-continuous uniformly with respect to $\varepsilon \in\left(0, \varepsilon_{0}\right]$, thus also for $\varepsilon=0$.

The proofs of Propositions 1.1.2-1.1.4 essentially use the fact that $f(s)$ is monotonically increasing.

Proposition 1.1.5. - For any given $g \in H_{-1}(U)$, the operator

$$
H_{1 / 2}(\partial U) \ni \phi_{\infty} \stackrel{\left(\mathscr{R}_{\varepsilon, \infty}^{\lambda}\right)^{-1}}{\longrightarrow} u_{\varepsilon, \infty}^{\lambda} \in H_{1}(U)
$$


is Hölder-continuous with exponent $\alpha=\frac{1}{2}$ uniformly with respect to $\varepsilon$.

Moreover, the following a priori estimate holds:

$$
\left\|\left(\mathfrak{H}_{\varepsilon, \infty}^{\lambda}\right)^{-1}\left(\phi_{1}, g\right)-\left(\mathfrak{Q}_{\varepsilon, \infty}^{\lambda}\right)^{-1}\left(\phi_{2}, g\right)\right\|_{H_{1}(U)} \leqslant C\left(\left\|\Phi_{1}-\Phi_{2}\right\|_{L_{2}(U)}^{1 / 2}+\left\|\Phi_{1}-\Phi_{2}\right\|_{H_{1}(U)}\right),
$$

where $\Phi_{i}, j=1,2$, is the solution of the linear problem

$$
\begin{cases}A_{\infty} \Phi_{j}=0, & x \in U \\ \pi_{0} \Phi_{j}=\phi_{j}, & x^{\prime} \in \partial U\end{cases}
$$

and the constant $C$ does not depend on $\varphi_{j}, g$ and $\varepsilon$.

Proof. - Let $u_{j}=\left(\mathfrak{H}_{\varepsilon, \infty}^{\lambda}\right)^{-1}\left(\phi_{j}, g\right)$ and denote $v_{j}=u_{j}-\Phi_{i}$, where $\Phi_{j}$ is defined by (1.1.5). Then $v=v_{1}-v_{2}$ is the solution of the problem:

$$
\begin{cases}A_{\infty} v+\lambda\left[f\left(\frac{v_{1}+\Phi_{1}}{\varepsilon}\right)-f\left(\frac{v_{2}+\Phi_{2}}{\varepsilon}\right)\right]=0, & x \in U, \\ \pi_{0} v\left(x^{\prime}\right)=0, & x^{\prime} \in \partial U .\end{cases}
$$

The inner produet in $L^{2}(U)$ after the integration by parts yields:

$$
\begin{aligned}
\int_{U} \sum a_{k j}^{\infty}(x) v_{x_{j}} v_{x_{k}} d x+\lambda \int_{U}\left[f\left(\frac{v_{1}+\Phi_{1}}{\varepsilon}\right)-f\left(\frac{v_{2}+\Phi_{2}}{\varepsilon}\right)\right]\left(\Phi_{1}-\Phi_{2}+v\right) d x= \\
=\lambda \int_{U}\left[f\left(\frac{v_{1}+\Phi_{1}}{\varepsilon}\right)-f\left(\frac{v_{2}+\Phi_{2}}{\varepsilon}\right)\right]\left(\Phi_{1}-\Phi_{2}\right) d x .
\end{aligned}
$$

The monotonicity of $f(s)$ leads to the inequality:

$$
\begin{aligned}
& \int_{U} \sum a_{k j}^{\infty}(x) v_{x_{j}} v_{x_{k}} d x \leqslant \lambda \int_{U}\left[f\left(\frac{u_{1}}{\varepsilon}\right)-f\left(\frac{u_{2}}{\varepsilon}\right)\right]\left(\Phi_{1}-\Phi_{2}\right) d x \leqslant \\
& \leqslant 2 \lambda \max \left\{f_{+\infty},\left|f_{-\infty}\right|\right\}(\text { meas } U)^{1 / 2}\left\|\Phi_{1}-\Phi_{2}\right\|_{\nu^{2}(E)} .
\end{aligned}
$$

As a consequence of (1.1.7), one gets the estimate:

$$
\|\nabla v\|_{L_{2}(U)}^{2} \leqslant 2 \lambda \mu^{-1} \max \left(f_{+\infty},\left|f_{-\infty}\right|\right\}(\operatorname{meas} U)^{1 / 2}\left\|\Phi_{1}-\Phi_{2}\right\|_{L_{2}(U)}
$$

where $\mu$ is the ellipticity constant for $A_{\infty}(x, \partial / \partial x)$. Hence,

$$
\|v\|_{H_{1}(U)} \leqslant C_{1}\left\|\Phi_{1}-\Phi_{2}\right\|_{L_{2}(U)}^{1 / 2}
$$

where $C_{1}$ depends only on $\lambda, \mu, f_{ \pm \infty}$, meas $U$ and the constant $v$ in the Poincaré's

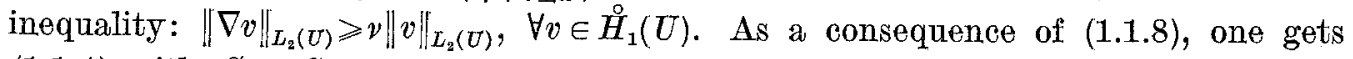
(1.1.4) with $O=O_{1}+1$. 


\subsection{Convergence for $\varepsilon \rightarrow 0$.}

In this section, an estimate for the $H_{1}$-norm of $u_{\varepsilon, \infty}^{\lambda}-u_{\infty}^{\lambda}$ is given, which is a slight generalization of the result established previously in $[16,2]$. Moreover, if the free boundary $\partial E_{0}\left(u_{\infty}^{\lambda}\right)$ is a sufficiently smooth manifold, it is proved that the $H_{1}$-norm of $u_{\varepsilon, \infty}^{\lambda}-u_{\infty}^{\lambda}$ is of order $O\left(\varepsilon^{3 / 4}\right)$ as $\varepsilon \downarrow 0$. It will be shown in the next section that there exists a critical value $\lambda_{c}\left(\phi_{\infty}, g_{\infty}\right)$ of $\lambda$ such that for $\lambda<\lambda_{c}$, the norm of $u_{\varepsilon, \infty}^{\lambda}-u_{\infty}^{\lambda}$ is of order $O(\varepsilon)$ as $\varepsilon \downarrow$.

THEOREM 1.2.1. - Under the regularity assumption

$$
\phi_{\infty} \in C^{2}(\partial U), \quad g_{\infty} \in C^{0}(\bar{U}) \text {, }
$$

the following estimate holds:

$$
\left\|u_{\varepsilon, \infty}^{\lambda}-u_{\infty}^{\lambda}\right\|_{H_{1}(U)} \leqslant C \varepsilon^{1 / 2},
$$

where the constant $C$ depends only on $\lambda, f, A_{\infty}$ and $U$.

Theorem 1.2.1 is proved by using the monotonicity of $f$ and the fact that the functions $u_{\varepsilon, \infty}^{\lambda}, u_{\infty}^{\lambda}$ can be characterized as solutions of corresponding elliptic variational inequalities.

Using a compactness argument and the uniqueness of the solution $u_{\infty}^{\lambda}$ to the reduced problem, one can also prove the following

Proposition 1.2.2. - If $g_{\infty} \in C^{0}(\bar{U}), \phi_{\infty} \in C^{2}(\partial U)$, then one has:

$$
\lim _{\varepsilon \rightarrow 0}\left\|u_{\varepsilon, \infty}^{\lambda}-u_{\infty}^{\lambda}\right\|_{\alpha^{1, \alpha}(\bar{\delta})}=0, \quad \forall \alpha \in[0,1) .
$$

Now we are going to establish the main result in this section.

Theorem 1.2.3. - Assume that $\phi_{\infty}>0$ on $\partial U, g_{\infty} \equiv 0$ in $U$, that the free boundary $\partial E_{0}\left(u_{\infty}^{\lambda}\right)$ is a $O^{4}$-manifold of dimension $n-1$, and that the function $f$ satisfies the condition (0.2). Then the following estimate holds:

$$
\left\|u_{\varepsilon, \infty}^{\lambda}-u_{\infty}^{\lambda}\right\|_{H_{1}\left(U^{\prime}\right.} \leqslant C \varepsilon^{3 / 4}
$$

where the constant $O$ does not depend on $\varepsilon$.

One constructs asymptotic solutions of the problem $\mathfrak{A}_{\varepsilon, \infty}^{\lambda}$ with a specially chosen piecewise linear function $f=f_{l}$ in order to prove this theorem. More precisely, introducing the function

$$
f_{l}(s)=\mathscr{H}(s) \min \left\{l s, f_{\infty}\right\}+\mathscr{H}(-s) \max \left\{l s, f_{-\infty}\right\}
$$


where $\mathscr{H}(s)$ is Heaviside's function and the parameter $l>0$ will be chosen later, one applies an appropriate modification of Vishik-Lyusternik's method $([19,20]$; see also $[16,18])$ for constructing asymptotic solutions of $\mathfrak{U}_{\varepsilon, \infty}^{\lambda}$ with $f(s)=f_{l}(s)$ and for establishing (1.2.4).

For $x$ in a sufficiently small neighbourhood of the free boundary $\partial E_{0}\left(u_{\infty}^{\lambda}\right)$, define $x^{\prime} \in \partial E_{0}\left(u_{\circ}^{\lambda}\right), \varrho \in \boldsymbol{R}$ by the relations:

$$
\begin{aligned}
& \left|x-x^{\prime}\right|=\min _{y^{\prime} \in \partial E_{0}\left(u^{\lambda}\right)}\left|x-y^{\prime}\right|=\operatorname{dist}\left(x, \partial E_{0}\left(u^{\lambda}\right)\right)=|\varrho|, \\
& \varrho>0 \quad \text { on } E_{+}\left(u^{\lambda}\right), \\
& \varrho \leqslant 0 \quad \text { on } E_{0}\left(u^{\lambda}\right) .
\end{aligned}
$$

If $x \in E_{+}\left(u^{\lambda}\right)$ and lies in the neighbourhood above (where the coordinates $\left(x^{\prime}, \varrho\right)$ are well defined), then the operator $A_{\infty}$ can be rewritten as follows:

$$
A_{\infty}\left(x, \frac{\partial}{\partial x}\right)=-\left(a(x) \frac{\partial^{2}}{\partial \varrho^{2}}+\left(b(x)+c(x) \nabla^{\prime}\right) \frac{\partial}{\partial \varrho}+B\left(x, \nabla^{\prime}\right)\right)
$$

where $\nabla^{\prime}$ denotes the gradient with respect to $x^{\prime} \in \partial E_{0}\left(u^{\lambda}\right)$ and where $B\left(x, \nabla^{\prime}\right)$ is a differential operator of second order with sufficiently smooth coefficients. Besides, the functions $a(x)>0, b(x), c(x)$ are sufficiently smooth, since the manifold $\partial E_{0}\left(u^{\lambda}\right)$ is supposed to be sufficiently smooth. Let $U_{\varepsilon, i}=\left\{x \in U:|\varrho|<2 \varepsilon^{1 / 3}\right\}$ and $U_{\varepsilon, e}=$ $=\left\{x \in U:|\varrho|>\varepsilon^{1 / 3}\right\}$ denote the interior and the exterior region, respectively. In $U_{\varepsilon, i}$, an asymptotic solution is sought in the form

$$
v_{\varepsilon}\left(x^{\prime}, \varrho\right)=\sum_{2 \leqslant j \leqslant 3} \varepsilon^{j / 2} v_{j}\left(x^{\prime}, \varepsilon^{-1 / 2} \varrho\right)
$$

where the functions $v_{j}, j=2,3$, are solutions of the following boundary value problems on $\boldsymbol{R}$ with $x^{\prime} \in \partial E_{0}\left(u^{\lambda}\right)$ playing the role of a parameter:

$$
\begin{cases}a\left(x^{\prime}\right) \frac{d^{2}}{d \zeta^{2}} v_{2}\left(x^{\prime}, \zeta\right)-\lambda f_{l}\left(v_{2}\left(x^{\prime}, \zeta\right)\right)=0, & \zeta \in \boldsymbol{R}, \\ v_{2}\left(x^{\prime}, \zeta\right)=o(1), & \zeta \rightarrow-\infty \\ v_{2}\left(x^{\prime}, \zeta\right)=\left(2 a\left(x^{\prime}\right)\right)^{-1} \lambda f_{\infty} \zeta^{2}+O(1), & \zeta \rightarrow+\infty\end{cases}
$$

$$
\begin{cases}a\left(x^{\prime}\right) \frac{d^{2}}{d \zeta^{2}} v_{3}\left(x^{\prime}, \zeta\right)-\lambda f_{l}^{\prime}\left(v_{2}\left(x^{\prime}, \zeta\right)\right) v_{3}\left(x^{\prime}, \zeta\right)=\gamma_{3}\left(x^{\prime}, \zeta\right), & \zeta \in \boldsymbol{R}, \\ v_{3}\left(x^{\prime}, \zeta\right)=o(1), & \zeta \rightarrow-\infty \\ v_{3}\left(x^{\prime}, \zeta\right)=\lambda f_{\infty}(1 / 6) \beta_{3}\left(x^{\prime}\right) \zeta^{3}+O(1), & \zeta \rightarrow+\infty\end{cases}
$$


with

$$
\begin{gathered}
\gamma_{3}\left(x^{\prime}, \zeta\right)=-\lambda \zeta\left(a\left(x^{\prime}\right)\right)^{-1} a_{\varrho}^{\prime}\left(x^{\prime}\right) f_{b}\left(v_{2}\left(x^{\prime}, \zeta\right)\right)-\left(b\left(x^{\prime}\right)+c\left(x^{\prime}\right) \nabla^{\prime}\right) v_{2 \zeta}, \\
\beta_{3}\left(x^{\prime}\right)=-\left(a\left(x^{\prime}\right)\right)^{-1} a_{\varrho}\left(x^{\prime}\right)+\left(b\left(x^{\prime}\right)+c\left(x^{\prime}\right) \nabla^{\prime}\right)\left(\left(a\left(x^{\prime}\right)\right)^{-1}\right) .
\end{gathered}
$$

The solution of (1.2.7) is given by

$$
v_{2}=v_{2}^{(l)}\left(x^{\prime}, \zeta\right)= \begin{cases}\frac{1}{l} \exp \left(\sqrt{\frac{\lambda f_{\infty} l}{a\left(x^{\prime}\right)}} \zeta\right)-1, & \zeta \leqslant \sqrt{\frac{a\left(x^{\prime}\right)}{\lambda f_{\infty} l}}, \\ \frac{\lambda f_{\infty}}{2 a\left(x^{\prime}\right)} \zeta^{2}+\frac{1}{2 l}, & \zeta>\sqrt{\frac{a\left(x^{\prime}\right)}{\lambda f_{\infty} l}},\end{cases}
$$

and, $v_{2 \zeta} \stackrel{\text { def }}{=}(d / d \zeta) v_{2}$ being a solution of the homogeneous differential the boundary value problem for $v_{3}$ can be solved using the variation of constants' method.

Let $\chi \in C_{0}^{\infty}(\boldsymbol{R})$ be a function which is identically one on the interval $[-1,1]$ and the support of $\chi$ be contained in $[-2,2]$. Let $z_{\varepsilon}$ be defined by

$$
z_{\varepsilon}(x)=\chi\left(\varepsilon^{-1 / 3} \varrho\right) v_{\varepsilon}\left(x^{\prime}, \varrho\right)+\left(1-\chi\left(\varepsilon^{-1 / 3} \varrho\right)\right) u_{\infty}^{2}(x) \text {. }
$$

Obviously, this function satisfies the boundary condition $\pi_{0} z_{\varepsilon}=\phi_{\infty}$ on $\partial U$.

Lemma 1.2.4. - There exist constants $C, \varepsilon_{0}$, such that

$$
\left\|A_{\infty} z_{\varepsilon}+\lambda f_{l}\left(\frac{z_{\varepsilon}}{\varepsilon}\right)\right\|_{L_{2}(\theta)} \leqslant C \varepsilon^{5 / 6}
$$

for $\varepsilon \in\left(0, \varepsilon_{0}\right]$.

PRoof. - We shall proceed by splitting the proof in several steps. Write $u^{\lambda}=u_{\infty}^{\lambda}$.

(i) For $x \in U_{\varepsilon, e}$, one has:

$$
u^{\lambda}(x) \geqslant p \varrho^{2} \geqslant p \varepsilon^{2 / 3}
$$

with a constant $p>0$. Thus, $\varepsilon^{-1} z_{\varepsilon}(x)=\varepsilon^{-1} u^{\lambda}(x) \geqslant p \varepsilon^{-1 / s}$ and $f_{l}\left(\varepsilon^{-1} z_{\delta}(x)\right) \equiv 1$ for $\varepsilon \in\left(0, \varepsilon_{0}\right], \varepsilon_{0}$ sufficiently small. Thus, $A_{\infty} z_{\varepsilon}+\lambda f_{l}\left(\varepsilon^{-1} z_{\varepsilon}\right) \equiv 0$ for $\varepsilon \in\left(0, \varepsilon_{0}\right], x \in U_{\varepsilon, \varepsilon}$.

(ii) It will be shown that

$$
\left\|f_{l}\left(v_{2}+\sqrt{\varepsilon} v_{3}\right)-\left(f_{l}\left(v_{2}\right)+\sqrt{\varepsilon} f_{l}^{\prime}\left(v_{2}\right) v_{3}\right)\right\|_{L^{2}\left(U_{\varepsilon, i}\right)} \leqslant C \varepsilon
$$

where $v_{j}=v_{j}\left(x^{\prime}, \varepsilon^{-1 / 2} \varrho\right)$. Without restriction of generality, one can assume that $\lambda=f_{\infty}=1$. Since $f_{l}$ is piecewise linear, the function on the left hand side of (1.2.10) 
is zero if the interval $\left(v_{2}+\sqrt{\varepsilon} v_{3}, v_{2}\right)$ does not contain $l^{-1}$. Let now

$$
S_{\varepsilon}=\left\{\left(x^{\prime}, \varrho\right): v_{2}+\sqrt{\varepsilon} v_{3}<l^{-1}<v_{2}\right\} .
$$

If $\left(x^{\prime}, \varrho\right) \in S_{\varepsilon}$, then $\varepsilon^{-1 / 2} \varrho>\zeta_{0}\left(x^{\prime}\right) \stackrel{\text { def }}{=} \sqrt{l^{-1} a\left(x^{\prime}\right)}$ and

$$
\frac{1}{2 a\left(x^{\prime}\right)} \frac{\varrho^{2}}{\varepsilon}+\frac{1}{2 l}+\sqrt{\varepsilon} v_{3}<l^{-1}<\frac{1}{2 a\left(x^{\prime}\right)} \frac{\varrho^{2}}{\varepsilon}+\frac{1}{2 l} .
$$

Since $\left|v_{3}(\zeta)\right|=O\left(\zeta^{\circ}\right), \zeta \rightarrow \infty$, one obtains

$$
\varrho^{2}-C Q^{3}<\varepsilon \cdot \zeta_{0}\left(x^{\prime}\right)^{2}<\varrho^{2}
$$

with a constant $C$. Thus, $\left|\varrho-\sqrt{\varepsilon} \zeta_{0}\left(x^{\prime}\right)\right| \leqslant C \varepsilon$. Since $l v_{2}\left(x^{\prime}, \zeta_{0}\left(x^{\prime}\right)\right)=1$, the following inequality holds for $x \in S_{\varepsilon}$ :

$$
\left|f_{l}\left(v_{2}+\sqrt{\varepsilon} v_{3}\right)-\left(f_{l}\left(v_{2}\right)+\sqrt{\varepsilon} f_{l}^{\prime}\left(v_{2}\right) v_{3}\right)\right|=\left|l\left(v_{2}+\sqrt{\varepsilon} v_{3}\right)-1\right| \leqslant C \varepsilon^{1 / 2} .
$$

The last inequality yields (1.2.10), since the measure of $S_{\varepsilon}$ is of order $O(\varepsilon)$.

(iii) It will be shown that

$$
\left\|A_{\infty} z_{\varepsilon}+\lambda\left(f_{l}\left(v_{2}\right)+f_{l}^{\prime}\left(v_{2}\right) \sqrt{\varepsilon} v_{3}\right)\right\|_{\sigma^{o}\left(\bar{U}_{\delta, t}\right)} \leqslant C_{\varepsilon}^{2 / 3} .
$$

Consider first the region $|\varrho|<\varepsilon^{1 / 3}$, where $\chi \equiv 1$. One has

$$
B\left(x, \nabla^{\prime}\right) \tilde{z}_{\varepsilon}=B\left(x, \nabla^{\prime}\right)\left(\varepsilon v_{2}+\varepsilon^{3 / 2} v_{3}\right)=O\left(\varepsilon^{2 / 3}\right) .
$$

Thus, with $\zeta=\varepsilon^{-1 / 2} \varrho$, one has

$$
A z_{\varepsilon}+\lambda\left(f_{l}\left(v_{2}\right)+\sqrt{\varepsilon} f_{l}^{\prime}\left(v_{2}\right) v_{3}\right)=r(x, \varepsilon)+O\left(\varepsilon^{2 / 3}\right),
$$

where

$$
\begin{aligned}
& r(x, \varepsilon)=-a\left(x^{\prime}\right) v_{2 \zeta \zeta}\left(x^{\prime}, \zeta\right)+\lambda f_{l}\left(v_{2}\right) \\
&+\sqrt{\varepsilon}\left(-a\left(x^{\prime}\right) v_{3 \zeta \zeta}\left(x^{\prime}, \zeta\right)+\lambda f^{\prime}\left(v_{2}\right) v_{3}-\zeta a_{e}\left(x^{\prime}\right) v_{2 \zeta \zeta}\left(x^{\prime}, \zeta\right)-\right. \\
&\left.-\left(b\left(x^{\prime}\right)+c\left(x^{\prime}\right) \nabla^{\prime}\right) v_{2 \zeta}\left(x^{\prime}, \frac{\varrho}{\sqrt{\varepsilon}}\right)\right)=O\left(\varepsilon^{2 / 3}\right)
\end{aligned}
$$

according to the constructions of $v_{j}$.

In the region $\varepsilon^{1 / 3}<|\varrho|<2 \varepsilon^{1 / 3}$, one obtains

$$
A z_{\varepsilon}+\lambda\left(f_{l}\left(v_{2}\right)+\sqrt{\varepsilon} f_{l}^{\prime}\left(v_{2}\right) v_{3}\right)=\sum_{0 \leqslant i \leqslant 2} r_{i}(x, \varepsilon)
$$


where $r_{i}, 0 \leqslant i \leqslant 2$, are given by

$$
\begin{aligned}
r_{0}(x, \varepsilon)= & r(x, \varepsilon)-\left(a\left(x^{\prime}\right)+\varrho a_{\varrho}\left(x^{\prime}\right)\right)(1-\chi)\left(u_{\varrho \varrho}^{\lambda}-\left(v_{2 \zeta \zeta}+\sqrt{\varepsilon} v_{3 \zeta \zeta}\right)\right)- \\
& -\left(b+c \nabla^{\prime}\right)(1-\chi)\left(u_{\varrho}^{\lambda}-\sqrt{\varepsilon} v_{2 \zeta}\right)+\chi B\left(\varepsilon v_{2}+\varepsilon^{3 / 2} v_{3}\right)+(1-\chi) B u^{\lambda}, \\
r_{1}(x, \varepsilon)= & -2 a\left(x^{\prime}\right) \varepsilon^{-1 / 3} \chi^{\prime}\left(\varepsilon^{-1 / 3} \varrho\right) \frac{\partial}{\partial \varrho}\left(\varepsilon v_{2}+\varepsilon^{3 / 2} v_{3}-u^{\lambda}\right)- \\
& -\left(b+e \nabla^{\prime}\right) \varepsilon^{1 / 3} \chi^{\prime}\left(\varepsilon^{-1 / 3} \varrho\right)\left(\varepsilon v_{2}+\varepsilon^{3 / 2} v_{3}-u^{\lambda}\right), \\
r_{2}(x, \varepsilon)= & -a(x) \varepsilon^{-2 / 3} \chi^{\prime \prime}\left(\varepsilon^{-1 / 3} \varrho\right)\left(\varepsilon v_{2}+\varepsilon^{3 / 2} v_{3}-u^{\lambda}\right) .
\end{aligned}
$$

Using the boundary conditions for $v_{j}$ and the asymptotic expansion

$$
u^{\lambda}\left(x^{\prime}, \varrho\right)=\left(2 a\left(x^{\prime}\right)\right)^{-1} \lambda f_{\infty} \varrho^{2}+\lambda f_{\infty}(1 / 6) \beta_{3}\left(x^{\prime}\right) \varrho^{3}+O\left(\varrho^{4}\right), \quad \varrho \downarrow 0,
$$

one checks easily that

$$
\sup _{\varepsilon^{1 / 3}<\varrho<2 \varepsilon^{1 / 3}}\left|r_{i}(x, \varepsilon)\right| \leqslant C \varepsilon^{2 / 3}, \quad i=0,1,2,
$$

where the constant $C$ does not depend upon $\varepsilon$. Thus (1.2.11) is proved.

(iv) Since $u^{\lambda}(x) \geqslant p \varrho^{2}$ for $x \in E_{+}\left(u^{\lambda}\right)$ with a constant $p>0$, one can choose $\varepsilon_{0}$ so small that for $\forall \varepsilon \in\left(0, \varepsilon_{0}\right], \forall x \in U_{\varepsilon, e}$,

$$
f\left(\varepsilon^{-1} z_{\varepsilon}(x)\right)=f_{\infty} \quad \text { and } \quad f\left(v_{2}\left(x^{\prime}, \varepsilon^{-1 / 2} \varrho\right)+\varepsilon^{1 / 2} v_{3}\left(x^{\prime}, \varepsilon^{-1 / 2} \varrho\right)\right)=f_{\infty}
$$

for $x \in U_{\varepsilon, i} \cap U_{\varepsilon, e}$. Thus

$$
\begin{aligned}
&\left\|A_{\infty} z_{\varepsilon}(x)+\lambda f_{l}\left(\varepsilon^{-1} z_{\varepsilon}(x)\right)\right\| \|_{L_{2}(U)}=\left\|A_{\infty} z_{\varepsilon}(x)+\lambda f_{l}\left(\varepsilon^{-1} z_{\varepsilon}(x)\right)\right\|_{L_{2}\left(U_{\varepsilon, i}\right)}= \\
&=\left\|A_{\infty} \tilde{w}_{\varepsilon}(x)+\lambda f_{l}\left(v_{2}+\varepsilon^{1 / 2} v_{3}\right)\right\|_{L_{3}\left(U_{s, i}\right)} \leqslant\left\|A_{\infty} z_{\varepsilon}+\lambda\left(f_{l}\left(v_{2}\right)+f_{l}^{\prime}\left(v_{2}\right) \sqrt{\varepsilon} v_{3}\right)\right\|_{L_{2}\left(U_{\varepsilon, i}\right)}+ \\
& \\
&+\lambda\left\|f_{l}\left(v_{2}\right)+f_{l}^{\prime}\left(v_{2}\right) \varepsilon^{1 / 2} v_{3}-f_{l}\left(v_{2}+\varepsilon^{1 / 2} v_{3}\right)\right\|_{L_{\varepsilon}\left(U_{s, l}\right)} \leqslant C \varepsilon^{5 / 6}
\end{aligned}
$$

as a consequence of $(1.2 .10),(1.2 .11)$ and given that meas $\left(U_{\varepsilon, i}\right)=O\left(\varepsilon^{1 / 3}\right), \varepsilon \downarrow 0$.

Lemma 1.2.4 is proved.

In order to prove Theorem 1.2.3 above, several anxiliary results will be needed.

Lemca 1.2.5. - There exists a well defined value of the parameter $l \in(0, \infty)$, such that

$$
I(l)=\int_{-\infty}^{\infty}\left(f\left(v_{2}^{(l)}(\zeta)\right)-f_{l}\left(v_{2}^{(l)}(\zeta)\right)\right) d \zeta=0
$$


L. S. FRANK - W. D. WENDT: Elliptic and parabolic singular, ete. 273

Proof. - Let $v(\zeta)=v_{\mathbf{2}}^{(\mathbf{1})}(\zeta)$. Then $v_{2}^{(l)}(\zeta)=l^{-1} v\left(l^{1 / 2} \zeta\right)$. Using the substitution $\eta=v(\sqrt{l} \zeta)$, one gets:

$$
\sqrt{ } \bar{l} I(l)=\int_{0}^{\infty}\left(f\left(l^{-1} \eta\right)-f_{1}(\eta)\right) \frac{d \eta}{v^{\prime}\left(v^{-1}(\eta)\right)}
$$

The right hand side is a strictly decreasing function of $l \in(0, \infty)$, so that $I(l)$ has at most one zero. For $l$ sufficiently large, one has $f\left(l^{-1} \eta\right)-f_{1}(\eta)<0, \forall \eta>0$, so that $I(l)<0$ for $l \gg 1$. Now $I(l)>0$ for $l$ sufficiently small. In fact, one has:

$$
\begin{aligned}
& \left|\int_{1}^{\infty}\left(f\left(l^{-1} \eta\right)-f_{1}(\eta)\right)\left(v^{\prime}\left(v^{-1}(\eta)\right)\right)^{-1} d \eta\right| \\
& =\left|\int_{1}^{\infty}\left(f\left(l^{-1} \eta\right)-f_{\infty}\right)\left(v^{\prime}\left(v^{-1}(\eta)\right)\right)^{-1} d \eta\right| \leqslant C l \int_{1}^{\infty} \eta^{-3 / 2} d \eta
\end{aligned}
$$

with $O$ independent of $l$. Hence,

$$
\sqrt{\imath} I(l)=\int_{0}^{1}\left(f\left(l^{-1} \eta\right)-f_{1}(\eta)\right)\left(v^{\prime}\left(v^{-1}(\eta)\right)\right)^{-1} d \eta+O(l), \quad \text { when } l \downarrow 0 .
$$

Therefore,

$$
\lim _{4 \downarrow 0} \sqrt{\imath} I(l)=\int_{0}^{1}\left(f_{\infty}-f_{1}(\eta)\right)\left(v^{\prime}\left(v^{-1}(\eta)\right)\right)^{-1} d \eta>0
$$

and Lemma 1.2.5 is proved.

We choose $l$ to be the zero of $I(l)$. Let $h(s)=f(s)-f_{l}(s)$, and for $a>0$, define $U_{a}=\left\{x \in U: \operatorname{dist}\left(x, \partial E_{0}\left(u_{\infty}^{\lambda}\right)\right)>a\right\}, R_{a}=\{\varrho \in \boldsymbol{R}:|\varrho|>a\}$.

We choose $a>0$ so small that for $|\varrho|<a$, the mapping $x \rightarrow\left(x^{\prime}, \varrho\right)$ is a diffeomorphism.

Lemma 1.2.6. - There exist constants $C, \varepsilon_{0}>0$, which do not depend upon $\varepsilon$ and such that for $\varepsilon \in\left(0, \varepsilon_{0}\right]$ holds:

$$
\begin{aligned}
& \text { (i) }\left\|h\left(\varepsilon^{-1} z_{\varepsilon}(x)\right)\right\| \|_{L_{2}\left(U_{a}\right)} \leqslant C \varepsilon ; \\
& \text { (ii) }\left\|h\left(v_{2}\left(x^{\prime}, \varepsilon^{-1 / 2} \varrho\right)\right)\right\|_{L_{2}\left(\partial E_{0} \times \mathbf{R}_{a}\right)} \leqslant C \varepsilon ; \\
& \text { (iii) }\left\|h\left(v_{2}\left(x^{\prime}, \varepsilon^{-1 / 2} \varrho\right)\right)\right\|_{H_{-1}\left(U \backslash U_{a}\right)} \leqslant C \varepsilon^{3 / 4} .
\end{aligned}
$$

Proof. - (i) For $x \in U_{a} \cap E_{+}\left(u_{\infty}^{\lambda}\right)$, one has: $z_{\varepsilon}(x)=u^{\lambda}(x) \geqslant p>0$, where $p$ does not depend on $\varepsilon, x$. The inequality $|h(s)| \leqslant C(1+|s|)^{-1}$ and the fact that for $x \in D_{a} \cap$ $\cap E_{0}\left(u_{\infty}^{\lambda}\right)$, one has: $z_{\varepsilon}(x) \equiv 0$, so that $h\left(\varepsilon^{-1} z_{\varepsilon}(x)\right)=0$ yield the first jpart of the Lemma. 
(ii) Since for $\varrho \in \boldsymbol{R}_{a}$ holds $v_{2}\left(x^{\prime}, \varepsilon^{-1 / 2} \varrho\right) \geqslant \varepsilon^{-1} p$, where $p$ does not depend on $x^{\prime}, \varepsilon$, the second inequality can be proved similarly to the first one.

(iii) Denote $t\left(x^{\prime}, s\right)=h\left(v_{2}\left(x^{\prime}, s\right)\right)$. One has .

$$
\left\|t\left(x^{\prime}, \varepsilon^{-1 / 2} \varrho\right)\right\|_{I_{-1}\left(\partial{E_{0}}_{0} \times(-a, 0)\right)} \leqslant\left\|t\left(x^{\prime}, \varepsilon^{-1 / 2} \varrho\right)\right\|_{H_{-1}\left(\partial E_{0} \times \boldsymbol{R}\right)}+\left\|t\left(x^{\prime}, \varepsilon^{-1 / 2} \varrho\right)\right\|_{I_{2}\left(\partial E_{0} \times \boldsymbol{R}_{\alpha}\right)} .
$$

Let $T\left(x^{\prime}, \zeta\right)=\int_{-\infty}^{\zeta} t\left(x^{\prime}, s\right) d s$. The inequality $\left|t\left(x^{\prime}, s\right)\right| \leqslant C\left(1+s^{2}\right)^{-1}$, which holds uniformly with respect to $x^{\prime} \in \partial E_{0}$, and Lemma 1.2.5 yield $\left|T\left(x^{\prime}, \zeta\right)\right| \leqslant C(1+|\zeta|)^{-1}$. Thus, $T \in L_{2}\left(\partial E_{0} \times \boldsymbol{R}\right)$ and

$$
\begin{aligned}
\left\|t\left(x^{\prime}, \varepsilon^{-1 / 2} \varrho\right)\right\|_{H_{-1}\left(\partial B_{0} \times \boldsymbol{R}\right)}=\sup _{\varphi \in H_{1}(U)}\|\varphi\|_{H_{1}(U)}^{-1}\left|\int t\left(x^{\prime}, \varepsilon^{-1 / 2} \varrho\right) \varphi\left(x^{\prime}, \varrho\right) d x^{\prime} d \varrho\right|= \\
\quad=\sup _{\varphi \in \bar{H}_{1}}\|\varphi\|_{H_{1}(U)}^{-1}\left|\int \varepsilon^{1 / 2} T\left(x^{\prime}, \varepsilon^{-1 / 2} \varrho\right) \frac{\partial \varphi}{\partial \varrho}\left(x^{\prime}, \varrho\right) d x^{\prime} d \varrho\right| \leqslant \\
\quad \leqslant \varepsilon^{1 / 2}\left\|T\left(x^{\prime}, \varepsilon^{-1 / 2} \varrho\right)\right\|_{L_{2}\left(\partial E_{0} \times \boldsymbol{R}\right)} \sup _{\varphi \in H_{1}(U)}\|\varphi\|_{H_{1}(U)}^{-1}\left\|\frac{\partial \varphi}{\partial \varrho}\right\|_{L_{2}(U)} \leqslant C \varepsilon^{3 / 4}
\end{aligned}
$$

As a consequence of (ii), the second term on the right hand side of (1.2.15) is of order $O(\varepsilon)$ and that ends the proof of Lemma 1.2.6.

LEMra 1.2.7. - (i) There exist constants $O, \varepsilon_{0}$, such that

$$
C^{-1}\left|v_{2}\left(\varepsilon^{-1 / 2} \varrho\right)\right| \leqslant \varepsilon^{-1}\left|z_{\varepsilon}(x)\right| \leqslant C\left|v_{2}\left(\varepsilon^{-1 / 2} \varrho\right)\right| \quad \forall \varrho \in(0, a), \quad \forall \varepsilon \in\left(0, \varepsilon_{0}\right] .
$$

(ii) There exists a constant $O>0$ such that

$$
\left\|h\left(\varepsilon^{-1} z_{\varepsilon}(x)\right)-h\left(v_{2}\left(\varepsilon^{-1 / 2} \varrho\right)\right)\right\|_{L_{9}\left(U \backslash U_{a}\right)} \leqslant \sigma^{3 / a} \quad \forall \varepsilon \in\left(0, \varepsilon_{0}\right] .
$$

Proof. - (i) For $0<\varrho<\varepsilon^{1 / 3}$, one has

$$
\varepsilon^{-1} z_{\varepsilon}(x)=v_{2}\left(\varepsilon^{-1 / 2} \varrho\right)\left(1+\left(v_{2}\left(\varepsilon^{-1 / 2} \varrho\right)\right)^{-1} \varepsilon^{1 / 2} v_{3}\left(\varepsilon^{-1 / 2} \varrho\right)\right) .
$$

The inequality

$$
\left|\left(v_{2}\left(\varepsilon^{-1 / 2} \varrho\right)\right)^{-1} \varepsilon^{1 / 2} v_{3}\left(\varepsilon^{-1 / 2} \varrho\right)\right| \leqslant C \varrho \leqslant C \varepsilon^{1 / 3}
$$

implies that (1.2.16) holds for $0<\varrho<\varepsilon^{1 / 3}$. Now let $\varepsilon^{1 / 3}<\varrho<2 \varepsilon^{1 / 3}$. Then

$$
\varepsilon^{-1} z_{\varepsilon}(x)=v_{2}\left(\varepsilon^{-1 / 2} \varrho\right)\left(1+r_{1}(\varepsilon, x)\right)
$$

where the function

$$
r_{1}(\varepsilon, x)=v_{2}\left(\varepsilon^{-1 / 2} \varrho\right)^{-1}\left[\chi\left(\varepsilon^{-1 / 3} \varrho\right) \varepsilon^{1 / 2} v_{3}+\left(1-\chi\left(\varepsilon^{-1 / 3} \varrho\right)\right)\left(\varepsilon^{-1} u^{\lambda}(x)-v_{2}\left(\varepsilon^{-1 / 2} \varrho\right)\right)\right]
$$


can be estimated as follows:

$$
\left|r_{1}(\varepsilon, x)\right| \leqslant C_{\varrho} \leqslant 2 C_{\varepsilon^{1 / 3}}, \quad \forall \varepsilon \in\left(0, \varepsilon_{0}\right]
$$

with some constant $C>0$.

Finally, let $2 \varepsilon^{1 / 3}<\varrho<a$. Then

$$
\varepsilon^{-1} z_{\varepsilon}(x)=\varepsilon^{-1} u^{\lambda}(x)=\varepsilon^{-1}\left(\left(2 a\left(x^{\prime}\right)\right)^{-1} \varrho^{2}+O\left(\varrho^{3}\right)\right)=v_{2}\left(\varepsilon^{-1 / 2} \varrho\right)\left(1+O\left(\left(v_{2}\left(\varepsilon^{-1 / 2} \varrho\right)\right)^{-1}\right)\right)
$$

and (1.2.16) is proved.

(ii) The left hand side of (1.2.17) can be estimated as follows:

$$
\begin{array}{r}
\left\|h\left(\varepsilon^{-1} z_{\delta}(x)\right)-h\left(v_{2}\left(\varepsilon^{-1 / 2} \varrho\right)\right)\right\| \|_{L_{2}\left(U \backslash V_{a}\right)}^{2} \leqslant \int_{|\varrho|<a}\left(\sup _{\theta \in\left(v_{2}, \varepsilon^{-1} z_{\varepsilon}\right)}\left|h^{\prime}(\theta)\right|\right)^{2}\left|\varepsilon^{-1} z_{\varepsilon}(x)-v_{2}\left(\varepsilon^{-1 / 2} \varrho\right)\right|^{2} d x \leqslant \\
\leqslant C \int_{0<\varrho<a}\left(\sup _{\theta \in\left(v_{2}, \varepsilon^{-1 z_{\varepsilon}}\right)}\left(1+\theta^{2}\right)^{-1}\right)^{2}\left|\varepsilon^{-1} z_{\varepsilon}(x)-v_{2}\left(\varepsilon^{-1 / 2} \varrho\right)\right|^{2} d x+ \\
+C \int_{-a<\varrho<0}\left|\varepsilon^{-1} z_{\varepsilon}(x)-v_{2}\left(\varepsilon^{-1 / 2} \varrho\right)\right|^{2} d x .
\end{array}
$$

Using (1.2.16) and the asymptotic behaviour of $v_{2}(\zeta)$ for $\zeta \rightarrow \infty$, one obtains:

$$
\begin{aligned}
& \left\|h\left(\varepsilon^{-1} z_{\varepsilon}(x)\right)-h\left(v_{2}\left(\varepsilon^{-1 / 2} \varrho\right)\right)\right\|_{L_{2}\left(U \backslash U_{\alpha}\right)}^{2} \leqslant \\
& \quad \leqslant C \int_{0<\varrho<a}\left(1+\left(\varepsilon^{-1 / 2} \varrho\right)^{4}\right)^{-2}\left(1+\varepsilon^{-1} \varrho^{3}\right)^{2} d \varrho+C \int_{-a<\varrho<0} \varepsilon\left|v_{3}\left(\varepsilon^{-1 / 2} \varrho\right)\right|^{2} d \varrho \leqslant O \varepsilon^{3 / 2} \quad \forall \varepsilon \in\left(0, \varepsilon_{0}\right],
\end{aligned}
$$

where $C$ does not depend upon $\varepsilon$, and that ends the proof of Lemma 1.2.7.

Proof of Theorem 1.2.3. - Let $l \in(0, \infty)$ be the zero of the function $I(l)$ defined by (1.2.13), and let $z_{\varepsilon}(x)$ be given by (1.2.9). One has:

$$
A_{\infty} z_{\varepsilon}+\lambda f_{l}\left(\varepsilon^{-1} z_{\varepsilon}(x)\right)=r_{\varepsilon}(x)
$$

where, according to Lemma 1.2.4,

$$
\left\|r_{\varepsilon}\right\|_{L_{2}(U)} \leqslant C \varepsilon^{5 / 6}, \quad \forall \varepsilon \in\left(0, \varepsilon_{0}\right]
$$

with some constant $C>0$ which does not depend on $\varepsilon$.

Writing the differential equations for $u_{\varepsilon}^{\lambda}$ and for $z_{s}$ and taking the difference, one gets for $u_{\varepsilon}^{\lambda}-z_{\varepsilon}$ :

$$
A_{\infty}\left(u_{\varepsilon}^{\lambda}-z_{\varepsilon}\right)+\lambda\left(f\left(\varepsilon^{-1} u_{\varepsilon}^{\lambda}(x)\right)-f_{l}\left(\varepsilon^{-1} \tilde{z}_{\varepsilon}(x)\right)\right)=-r_{\varepsilon}(x)-\lambda\left(f\left(\varepsilon^{-1} z_{\varepsilon}(x)\right)-f_{l}\left(\varepsilon^{-1} z_{\varepsilon}(x)\right)\right) .
$$


Taking the inner product with $u_{\varepsilon}^{\lambda}-z_{s}$ in $L_{2}(U)$ in the last equation and using the monotonicity of $f$, one gets the following estimates:

$$
\int_{U}\left(u_{\varepsilon}^{\lambda}-z_{\varepsilon}\right) A_{\infty}\left(u_{\varepsilon}^{\lambda}-z_{\varepsilon}\right) d x \leqslant\left(\left\|r_{\varepsilon}\right\|_{H_{-1}(U)}+\lambda\left\|f\left(\varepsilon^{-1} z_{\varepsilon}(x)\right)-f_{l}\left(\varepsilon^{-1} z_{\varepsilon}(x)\right)\right\|_{H_{-1}(U)}\right)\left\|u_{\varepsilon}^{\lambda}-z_{\varepsilon}\right\|_{H_{1}(U)} .
$$

The integration by parts and Poincaré's Lemma yield:

$$
\left\|u_{\varepsilon}^{\lambda}-z_{\varepsilon}\right\|_{H_{1}(U)} \leqslant C\left(\left\|r_{\varepsilon}\right\|_{H_{-1}(U)}+\left\|f\left(\varepsilon^{-1} z_{\varepsilon}(x)\right)-f_{l}\left(\varepsilon^{-1} z_{\varepsilon}(x)\right)\right\|_{H_{-1}(U)}\right) .
$$

The last inequality is also a consequence of Proposition 1.1.3.

According to (1.2.18), the first term on the right hand side is bounded by $C \varepsilon^{5 / 6}$.

According to Lemmas 1.2.6, 1.2.7, the second term can be estimated as follows:

$$
\begin{aligned}
\left\|f\left(\varepsilon^{-1} z_{\varepsilon}(x)\right)-f_{l}\left(\varepsilon^{-1} z_{\varepsilon}(x)\right)\right\|_{H_{-1}(U)}=\| & h\left(\varepsilon^{-1} z_{\varepsilon}(x)\right) \|_{H_{-1}(U)} \leqslant \\
& \leqslant\left\|h\left(\varepsilon^{-1} z_{\varepsilon}(x)\right)\right\|_{H_{-1}\left(U_{a}\right)}+\left\|h\left(\varepsilon^{-1} z_{\varepsilon}(x)\right)\right\|_{H_{-1}\left(U \backslash U_{a}\right)} \leqslant \\
& \leqslant\left\|h\left(\varepsilon^{-1} z_{\varepsilon}(x)\right)\right\|_{L_{z}\left(U_{a}\right)}+\left\|h\left(v_{2}\left(x^{\prime}, \varepsilon^{-1 / 2} \varrho\right)\right)\right\|_{H_{-1}\left(U \backslash U_{a}\right)}+ \\
& +\left\|h\left(\varepsilon^{-1} z_{\varepsilon}(x)\right)-h\left(v_{z}\left(x^{\prime}, \varepsilon^{-1 / 2} \varrho\right)\right)\right\|_{H_{-1}\left(U \backslash U_{a}\right)} \leqslant \delta^{3 / 4}
\end{aligned}
$$

This ends the proof of Theorem 1.2.3.

REMARK 1.2.8. - Consider the problem $\mathfrak{H}_{\varepsilon, \infty}^{\lambda}$ in $U=(-1,1) \subset \boldsymbol{R}$ with $f=f_{l}$ defined in (1.2.5) and $A_{\infty}=-(d / d x)^{2}, g_{\infty} \equiv 0, \phi_{\infty}\left(x^{\prime}\right)=1$. The function $u_{\infty}^{\lambda}(x)=$ $=(\lambda / 2)(|x|-\xi)_{+}^{2}, \xi=1-\left(2 \lambda^{-1}\right)^{1 / 2}$, is the solution of the reduced problem $\mathfrak{A}_{\infty}^{\lambda}$. Let $v_{2}$ be the solution of (1.2.7) and let

$$
z_{\varepsilon}(x)=u_{\infty}^{\lambda}(x)(1-\chi(|x|-\xi))+\varepsilon v_{2}(\sqrt{\lambda / \varepsilon}(|x|-\xi)) \chi(|x|-\xi) .
$$

Similarly to the proof of Lemma 1.2.4, one checks that

$$
\left\|-z_{\varepsilon}^{\prime \prime}+\lambda f_{l}\left(\varepsilon^{-1} z_{\varepsilon}\right)\right\|_{\sigma^{\circ}(\bar{U})} \leqslant C \varepsilon
$$

where the constant $C$ does not depend on $\varepsilon$. Further, $\pi_{0} z_{\varepsilon}=1$. Partial integration yields:

$$
\left\|u_{\varepsilon, \infty}^{\lambda}-z_{\varepsilon}\right\|_{H_{1}(U)} \leqslant C \varepsilon,
$$

where the constant $C$ does not depend on $\varepsilon$.

Since $f_{l}^{\prime}(+0)>0$, the function $v_{2}(\zeta)$ and its derivative decrease exponentially for $\zeta \rightarrow-\infty$. Thus,

$$
\gamma \varepsilon^{3 / 4} \leqslant\left\|\tilde{z}_{\varepsilon}\right\|_{H_{1}(-\xi, \xi)} \quad \forall \varepsilon \in\left[0, \varepsilon_{0}\right],
$$


where the constant $\gamma>0$ does not depend on $\varepsilon$. The inequalities (1.2.21), (1.2.22) yield:

$$
\begin{array}{r}
\left\|u_{\varepsilon, \infty}^{\lambda}-u_{\infty}^{\lambda}\right\|_{H_{1}(U)} \geqslant\left\|u_{\varepsilon, \infty}^{\lambda}-u_{\infty}^{\lambda}\right\|_{H_{1}(-\xi, \xi)} \geqslant\left\|u_{\varepsilon, \infty}^{\lambda}\right\|_{H_{1}(-\xi, \xi)} \geqslant\left\|z_{\varepsilon}\right\|_{H_{1}(-\xi, \xi)}-\left\|u_{\varepsilon, \infty}^{\lambda}-z_{\varepsilon}\right\|_{H_{1}(U)} \geqslant \\
\gamma \varepsilon^{3 / 4}-C \varepsilon \geqslant \frac{\gamma}{2} \varepsilon^{3 / 4}, \quad \forall \varepsilon \in\left(0, \varepsilon_{0}\right],
\end{array}
$$

where $\varepsilon_{0}>0$ is sufficiently small. Thus, one has the following two-sided error estimate:

$$
C^{-1} \varepsilon^{3 / 4} \leqslant\left\|u_{\varepsilon, \infty}^{\lambda}-u_{\infty}^{\lambda}\right\|_{H_{1}(U)} \leqslant C \varepsilon^{3 / 4}
$$

with some constant $C>0$ which does not depend on $\varepsilon$. It can be shown that the estimate (1.2.23) holds in the general case, as well, if the assumptions of Theorem 1.2.3 and the condition $f^{\prime}(+0)>0$ are satisfied.

REMARK 1.2.9. - If $g_{\infty} \neq \equiv$, then the same argument with corresponding slight modifications in the construction of the asymptotic solutions of the problem $\mathfrak{A}_{\varepsilon, \infty}^{\lambda}$ with $f=f_{l}$ leads to the same estimate under the assumptions of Theorem 1.2.3.

\subsection{The critical value $\lambda_{\mathrm{c}}$ of $\lambda$.}

If $\phi_{\infty}\left(x^{\prime}\right) \neq 0, \forall x^{\prime} \in \partial U$, then some critical value $\lambda_{c}$ of the parameter $\lambda$ plays a special role in the investigation of the boundary value problem $\mathfrak{A}_{\infty}^{\lambda}$. Namely, if $\phi_{\infty}>0$, then for $\lambda<\lambda_{c}$ the problem $\mathfrak{H}_{\infty}^{\lambda}$ becomes linear, whereas for $\lambda>\lambda_{c}$ it is a nonlinear problem with piecewise constant discontinuous (across $\left.\partial E_{0}\left(u_{\infty}^{\lambda}\right)\right)$ nonlinearity. Denote by $C_{+}^{0}(\partial U)$ and $C_{-}^{0}(\partial U)$ the cones of continuous positive and negative functions on $\partial U$, respectively. Further, let $G(x, y)$ be Green's function for $A_{\infty}(x, \partial / \partial x)$ in $U$ with Dirichlet boundary conditions on $\partial U$ and denote by $E\left(x, y^{\prime}\right)$ the Poisson kernel for the Dirichlet problem for the equation $A_{\infty} u=0$.

Theorem 1.3.1. - (i) If $\phi_{\infty} \in C_{+}^{0}(\partial U)$ (respectively, $\phi_{\infty} \in C_{-}^{0}(\partial U)$ ), then there exists a well defined critical value $\lambda_{c}=\lambda_{c}^{+}\left(\phi_{\infty}, g_{\infty}\right)$ (respectively, $\lambda_{c}=\lambda_{c}^{-}\left(\phi_{\infty}, g_{\infty}\right)$ ), such that

$$
E_{0}\left(u_{\infty}^{\lambda}\right)=\emptyset \quad \text { if } \lambda<\lambda_{c},
$$

meas $\left(E_{0}\left(u_{\infty}^{\lambda}\right) \cap E_{-}\left(u_{\infty}^{\lambda}\right)\right)>0$ if $\lambda>\lambda_{c}\left(\right.$ respectively, meas $\left(E_{0}\left(u_{\infty}^{\lambda}\right) \cap E_{+}\left(u_{\infty}^{\lambda}\right)\right)>0$ if $\left.\lambda>\lambda_{c}\right)$.

(ii) If $\lambda_{c} \geqslant \lambda$ then $u_{\infty}^{\lambda}$ is the solution of the linear problem

$$
\begin{cases}A_{\infty}\left(x, \frac{\partial}{\partial x}\right) v_{\infty}^{\lambda}(x)=g_{\infty}(x)-\lambda f_{\infty}, & x \in U, \\ \pi_{0} v_{\infty}^{\lambda}(x)=\phi_{\infty}\left(x^{\prime}\right), & x^{\prime} \in \partial U .\end{cases}
$$

If $g_{\infty} \equiv 0$, then meas $E_{0}\left(u_{\infty}^{\lambda_{c}}\right)=0$. 
(iii) The functionals $\lambda_{c}^{+}, \lambda_{c}^{-}$can be represented as follows:

$$
\lambda_{c}^{ \pm}\left(\phi_{\infty}, g_{\infty}\right)=\min _{x \in D} \Lambda_{\phi_{\infty}, \theta_{\infty}}^{ \pm}(x),
$$

where the function $\Lambda_{\phi_{\infty}, s_{\infty}}^{ \pm}$is defined by:

$$
\Lambda_{\phi_{\infty}, g_{\infty}}^{+}(x)=\left(f_{ \pm \infty} \int_{U} G(x, y) d y\right)^{-1}\left(\int_{\partial U} E\left(x, y^{\prime}\right) \phi_{\infty}\left(y^{\prime}\right) d \sigma_{y^{\prime}}+\int_{U} G(x, y) g_{\infty}(y) d y\right) .
$$

The proof of Theorem 1.3.1 is similar to the proof of Theorem 2 in [9].

Proposition 1.3.2. - The functional $\left(\phi_{\infty}, g_{\infty}\right) \rightarrow \lambda_{c}^{+}\left(\phi_{\infty}, g_{\infty}\right)$ has the following properties:

(i) $\lambda_{c}^{+}\left(\alpha \phi_{\infty}, \alpha g_{\infty}\right)=\alpha \lambda_{c}^{+}\left(\phi_{\infty}, g_{\infty}\right), \forall \alpha>0$;

(ii) $\lambda_{c}^{+}\left(\phi_{\infty}^{(1)}, g_{\infty}^{(1)}\right) \leqslant \lambda_{c}^{+}\left(\phi_{\infty}^{(2)}, g_{\infty}^{(2)}\right)$, if $0 \leqslant \phi_{\infty}^{(1)} \leqslant \phi_{\infty}^{(2)}, g_{\infty}^{(1)} \leqslant g_{\infty}^{(2)}$;

(iii) $\lambda_{c}^{+}\left(\gamma \phi_{\infty}^{(1)}+(1-\gamma) \phi_{\infty}^{(2)}, \gamma g_{\infty}^{(1)}+(1-\gamma) g_{\infty}^{(2)}\right) \geqslant \gamma \lambda_{c}^{+}\left(\phi_{\infty}^{(1)}, g_{\infty}^{(1)}\right)+$

$$
+(1-\gamma) \lambda_{c}^{+}\left(\phi_{\infty}^{(2)}, g_{\infty}^{(2)}\right), \forall \gamma \in[0,1] \text {. }
$$

Proof. - One proves (i)-(iii), using the formula (1.3.2) for $\lambda_{c}^{+}\left(\phi_{\infty}, g_{\infty}\right)$.

Analogous properties has the functional $\lambda_{c}^{-}\left(\phi_{\infty}, g_{\infty}\right)$.

For $\lambda<\lambda_{c}$, the convergence result given in section 1.2 can be improved. One has the

THEOREM 1.3.3. - Assume that $\phi_{\infty} \in C_{+}^{0}(\partial U)$ and that $\lambda_{c}>\lambda$. Then the following estimate holds:

$$
\left\|u_{\varepsilon, \infty}^{\lambda}-u_{\infty}^{\lambda}\right\|_{W_{2}, p(U)} \leqslant C \varepsilon\left(\lambda_{c}-\lambda\right)^{-1}, \quad \forall \varepsilon>0, \forall p, 1<p<\infty
$$

where the constant $C$ depends only upon $p, L, U, A_{\infty}, \phi_{\infty}$ and $g_{\infty}$.

Proof. - Since $\mathfrak{A}_{\infty}^{\lambda}$ for $\lambda<\lambda_{c}$ is linear, the function $w=u_{\varepsilon, \infty}^{\lambda}-u_{\infty}^{\lambda}$ is the solution of the problem:

$$
\begin{cases}A_{\infty} w(x)=\lambda\left[f_{+\infty}-f\left(\frac{u_{\varepsilon, \infty}^{\lambda}}{\varepsilon}\right)\right], & x \in U, \\ \pi_{0} w\left(x^{\prime}\right)=0, & x^{\prime} \in \partial U .\end{cases}
$$

One can write:

$$
u_{\infty}^{\lambda}(x)=u^{\lambda_{e}}(x)+\left(\lambda_{\theta}-\lambda\right) v(x), \quad \lambda \leqslant \lambda_{0}
$$


where $v(x)$ is the solution of the problem

$$
\begin{cases}A_{\infty} v(x)=1, & x \in U \\ \pi_{0} v\left(x^{\prime}\right)=0, & x^{\prime} \in \partial U\end{cases}
$$

Since $v(x)>0, \forall x \in U$ and $u^{\lambda_{c}}\left(x^{\prime}\right)>0, \forall x^{\prime} \in \partial U$, one can find a constant $\gamma>0$ such that the sets

$$
U_{1}=\left\{x \in U: u^{\lambda_{c}}(x) \geqslant \gamma\left(\lambda_{c}-\lambda\right)\right\}, \quad U_{2}=\left\{x \in U: v(x) \geqslant \gamma\left(\lambda_{c}-\lambda\right)\right\}
$$

cover $\bar{U}$, so that one has:

$$
u_{\infty}^{\lambda}(x) \geqslant \gamma\left(\lambda_{c}-\lambda\right), \quad \forall x \in \bar{U}
$$

Of course, $\gamma$ depends upon $\phi_{\infty}$ and $g_{\infty}$.

Denote $v_{\varepsilon}(x)=\partial u_{\varepsilon, \infty}^{\lambda} / \partial \varepsilon$. Then $v_{\varepsilon}$ is the solution of the problem:

$$
\begin{array}{ll}
A_{\infty} v_{\varepsilon}+\frac{\lambda}{\varepsilon} f^{\prime}\left(\frac{u_{\varepsilon, \infty}^{\lambda}}{\varepsilon}\right) v_{\varepsilon}=\frac{1}{\varepsilon^{2}} f^{\prime}\left(\frac{u_{\varepsilon, \infty}^{\lambda}}{\varepsilon}\right) u_{\varepsilon_{1} \infty}^{\lambda}, & x \in U, \\
\pi_{0} v_{\varepsilon}\left(x^{\prime}\right)=0, & x^{\prime} \in \partial U .
\end{array}
$$

Since $f^{\prime}(s) \geqslant 0, u_{\varepsilon, \infty}^{\lambda} \geqslant 0$ for $\varepsilon$ sufficiently small (because $u_{\infty}^{\lambda}>0, \forall x \in \bar{U}, \forall \lambda<\lambda_{c}$ ), one gets the conclusion that $v_{\varepsilon}(x) \geqslant 0$, so that $u_{\varepsilon, \infty}^{\lambda}$ is monotonically increasing function of $\varepsilon$ and, in particular, one has:

$$
u_{\varepsilon, \infty}^{\lambda}(x) \geqslant u_{\infty}^{\lambda}(x) \geqslant \gamma\left(\lambda_{0}-\lambda\right), \quad \forall x \in \bar{U}, \varepsilon>0 .
$$

Hence,

and

$$
0 \leqslant f_{+\infty}-f\left(\frac{u_{\varepsilon, \infty}^{\lambda}}{\varepsilon}\right)=\int_{u_{\varepsilon, \infty}^{\lambda} / \varepsilon} f^{\prime}(s) d s \leqslant L \frac{\varepsilon}{\varepsilon+u_{\varepsilon, \infty}^{\lambda}} \leqslant \frac{L}{\gamma\left(\lambda_{c}-\lambda\right)} \varepsilon
$$

$$
\left\|f_{+\infty}-f\left(\frac{u_{\varepsilon, \infty}^{\lambda}}{\varepsilon}\right)\right\|_{L_{p}(U)} \leqslant \frac{L}{\gamma} \varepsilon\left(\lambda_{c}-\lambda\right)^{-1}(\text { meas } U)^{1 / p}
$$

As a consequence of the a priori estimates for second order linear elliptic operators, one gets, using (1.3.5), (1.3.6), the estimate (1.3.4).

COROLLARY 1.3.4. - If $\lambda_{o}-\lambda=\varepsilon^{\theta}, 0<\theta<1$, then $u_{\varepsilon, \infty}^{\lambda}$ converges to $u_{\infty}^{\lambda_{c}}$ in $W_{2, p}(U), \forall p<\infty$, as $\varepsilon \rightarrow 0$.

CoRollakX 1.3.5. - If $\lambda_{c}-\lambda=\varepsilon^{\theta}, 0<\theta<1$, then $u_{\varepsilon, \infty}^{\lambda}$ 'converges to $u_{\infty}^{\lambda_{c}}$ in $H_{1}(U)$ and the rate of convergence is $O\left(\varepsilon^{\theta}\right)$. In fact, let $\mu=\lambda_{c}-\lambda^{1-\theta}$. Then, using 
Proposition 1.1.4 and Theorem 1.3.3, one gets:

$$
\left\|u_{\varepsilon, \infty}^{\lambda}-u_{\infty}^{\lambda_{c}}\right\|_{H_{1}} \leqslant\left\|u_{\varepsilon, \infty}^{\lambda}-u_{\varepsilon, \infty}^{\mu}\right\|_{H_{1}}+\left\|u_{\varepsilon, \infty}^{\mu}-u_{\infty}^{\lambda_{c}}\right\|_{H_{1}} \leqslant C\left(|\lambda-\mu|+C \varepsilon\left(\lambda_{c}-\mu\right)^{-1}\right) \leqslant C \varepsilon^{\theta} .
$$

\subsection{Estimates for the critical value of $\lambda$.}

Let $G(x, y)$ denote Green's function for the operator $A_{\infty}(x, \partial / \partial x)$ with homogeneous Dirichlet boundary conditions and let $E\left(x, y^{\prime}\right)$ be the Poisson kernel for the Dirichlet problem for the equation $A_{\infty} u=0$. Denote by $C_{+}^{0}(U)$ and $C_{+}^{0}(\partial U)$ the cone of positive continuous functions on $U$ and $\partial U$, respectively. For functions $g \in C_{+}^{\mathbf{0}}(U)$ and $\phi \in C_{+}^{0}(\partial U)$, let $\mathscr{N}_{t}(g)$ and $\mathcal{H}_{t}(\phi)$ be their mean values of order $t$ :

F.

$$
\begin{aligned}
\mathcal{M}_{t}(g)=\left(\frac{1}{\operatorname{meas}(\bar{U})} \int_{U} g(x)^{t} d x\right)^{1 / t}, & & \forall t \in \boldsymbol{R}, \\
\mathcal{M}_{t}(\phi)=\left(\frac{1}{\operatorname{meas}(\partial \bar{U})} \int_{\partial U} \phi\left(x^{\prime}\right)^{t} d \sigma_{x^{\prime}}\right)^{1 / t}, & & \forall t \in \boldsymbol{R}, \\
\mathcal{M}_{t}(\phi)=\left(\frac{1}{2} \sum_{x^{\prime} \in \partial U U} \phi\left(x^{\prime}\right)^{t}\right)^{1 / t}, & & \forall t \in \boldsymbol{R}, \text { if } n=1 .
\end{aligned}
$$

In this section, it is assumed that $g_{\infty} \in C_{+}^{0}(U), \phi_{\infty} \in C_{+}^{0}(\partial U)$. For the investigation of the functional $\lambda_{o}\left(\phi_{\infty}, g_{\infty}\right)$, the assumption of positivity of $g_{\infty}$ is not a restriction of the generality. Indeed, (1.3.2) implies that

$$
\lambda_{c}^{+}\left(\phi_{\infty}, g_{\infty}\right)=\lambda_{c}^{+}\left(\dot{\phi}_{\infty}, g_{\infty}+\varrho\right)-\left(f_{\infty}\right)^{-1} \varrho, \quad \forall \varrho \in \boldsymbol{R} \text {. }
$$

Let

$$
v(x)=\int_{U} G(x, y) d y
$$

and let $\Sigma$ be the set of the points where the function $v$ attains a global maximum. For functions $g \in C_{+}^{0}(U), \phi \in C_{+}^{0}(\partial U)$, and for $x_{0} \in \Sigma$, define the mean values $\mathcal{N}_{x_{0}}(g)$, $\mathcal{N}_{x_{\mathrm{o}}}(\phi)$ as follows:

One has

$$
\begin{aligned}
& \mathcal{P}_{x_{0}}(g)=\left(v\left(x_{0}\right)\right)^{-1} \int_{U} G\left(x_{0}, y\right) g(y) d y, \\
& \mathcal{P}_{x_{0}}(\phi)=\int_{\partial U} E\left(x_{0}, y^{\prime}\right) \phi\left(y^{\prime}\right) d \sigma_{y^{\prime}} .
\end{aligned}
$$

Proposition 1.4.1. - Let $U \subset \boldsymbol{R}^{n}, n \geqslant 2$. Fof $\forall\left(\phi_{\infty}, g_{\infty}\right) \in O_{+}^{0}(\partial U) \times C_{+}^{0}(U), \forall t>0$, the following estimate holds:

$$
\varrho \cdot \mathcal{H}_{1-n / 2}\left(\phi_{\infty}\right)+\varrho_{t} \cdot \mathcal{H}_{-t}\left(g_{\infty}\right) \leqslant \lambda_{c}^{+}\left(\phi_{\infty}, g_{\infty}\right) \leqslant\left(f_{\infty}\right)^{-1} \min _{x_{0} \in \Sigma}\left(\left(v\left(x_{0}\right)\right)^{-1} \mathcal{N}_{x_{0}}\left(\phi_{\infty}\right)+\mathcal{N}_{x_{0}}\left(g_{\infty}\right)\right),
$$


where the constants $\varrho, \varrho_{t}$ are given by the formulae:

$$
\begin{aligned}
& \varrho=\left(f_{\infty}\right)^{-1} \operatorname{meas}(\partial U) \cdot \min _{x \in U} \mathcal{H}_{(n-2) / n}\left((v(x))^{-1} E(x, \cdot)\right), \\
& \varrho_{t}=\left(f_{\infty}\right)^{-1} \operatorname{meas}(U) \cdot \min _{x \in U} \mathscr{M}_{t^{\prime}(t+1)}\left((v(x))^{-1} G(x, \cdot)\right) .
\end{aligned}
$$

Proof. - The second part of (1.4.2) is obtained by estimating the maximum in (1.3.3) by the value of $A_{\phi_{\infty}, \theta_{\infty}}^{+}$at $x=x_{0}$.

In order to prove the first part of (1.4.2), note that for positive functions $h_{1}, h_{2}$, and for $p>1, p^{-1}+q^{-1}=1$, Hölder's inequality can be rewritten as follows:

$$
\int h_{1} \geqslant\left(\int h_{1}^{1 / p} h_{2}\right)^{p}\left(\int h_{2}^{a}\right)^{-p / q}
$$

One has:

$$
\begin{aligned}
f_{\infty} \lambda_{c}^{+}\left(\phi_{\infty}, g_{\infty}\right)= & \min _{x \in U}\left((v(x))^{-1} \int_{\partial U} E\left(x, y^{\prime}\right) \phi_{\infty}\left(y^{\prime}\right) d \sigma_{y^{\prime}}+(v(x))^{-1} \int_{U} G(x, y) g_{\infty}(y) d y\right) \geqslant \\
& \geqslant \min _{x \in U}\left((v(x))^{-1} \int_{\partial U} E\left(x, y^{\prime}\right) \phi_{\infty}\left(y^{\prime}\right) d \sigma_{y^{\prime}}\right)+\min _{x \in U}\left((v(x))^{-1} \int_{U} G(x, y) g_{\infty}(y) d y\right) .
\end{aligned}
$$

In order to estimate the first term from below, one applies (1.4.3) with $p=(n-2)^{-1} n$ and

$$
h_{1}\left(y^{\prime}\right)=(v(x))^{-1} E\left(x, y^{\prime}\right) \phi_{\infty}\left(y^{\prime}\right), \quad h_{2}\left(y^{\prime}\right)=\left(\phi_{\infty}\left(y^{\prime}\right)\right)^{-1 / p}
$$

The second term can be estimated similarly, with $p=t^{-1}(t+1)$ and

$$
h_{1}(y)=(v(x))^{-1} G(x, y) g_{\infty}(y), \quad h_{2}(y)=\left(g_{\infty}(y)\right)^{-1 / p} .
$$

This yields the elaim (1.4.2).

For the rest of this section, $U$ is assumed to be the unit ball in $\boldsymbol{R}^{n}$. If one has:

$$
\begin{gathered}
A_{\infty}\left(x, \frac{\partial}{\partial x}\right)=-\Delta, \quad f_{0}(s)=\operatorname{sgn} s, \\
g_{\infty}(x)=0,
\end{gathered}
$$

the estimate (1.4.2) takes the following form:

$$
2 n \mathcal{M} \mathcal{M}_{1-n / 2}\left(\phi_{\infty}\right) \leqslant \lambda_{e}^{+}\left(\phi_{\infty}, 0\right) \leqslant 2 n \mathcal{M}_{1}\left(\phi_{\infty}\right)
$$


Indeed, in this case, the number $\varrho$ can be computed as follows:

$$
\begin{aligned}
& \varrho=\min _{|x|<1} 2 n\left(\Omega_{n}\right)^{-1} \int_{\left|y^{\prime}\right|=1}\left|x-y^{\prime}\right|^{2-n} d \sigma_{y^{\prime}}=\min _{|x|<1} 2 n=2 n \quad \text { if } n \geqslant 3, \\
& \varrho=\min _{|x|<1} 4\left(\Omega_{2}\right)^{-1} \int_{\left|y^{\prime}\right|=1} \ln \left|x-y^{\prime}\right| d \sigma_{y^{\prime}}=\min _{|x|<1} 4=2 n \quad \text { if } n=2,
\end{aligned}
$$

where $\Omega_{n}$ denotes the surface of the unit ball in $\boldsymbol{R}^{n}$.

The following result shows that estimate (1.4.6) is sharp.

Proposition 1.4.2. - (i) If $n \geqslant 2$, then there exist nonconstant functions $\phi_{j} \in C_{+}^{0}(\partial U), j=1,2$, such that

$$
\lambda_{c}^{+}\left(\phi_{1}, 0\right)=2 n \mathcal{H}_{1-n / 2}\left(\phi_{1}\right), \quad \lambda_{o}^{+}\left(\phi_{2}, 0\right)=2 n \mathcal{M} \mathcal{H}_{1}\left(\phi_{2}\right) .
$$

(ii) There are no positive constants $\varepsilon_{n}$ and $\delta_{n}$ such that

$$
2 n \cdot \mathcal{M}_{1-n / 2+\varepsilon_{n}}(\phi) \leqslant \lambda_{c}^{+}(\phi, 0) \quad \forall \phi \in C_{+}^{0}(\partial U)
$$

or

$$
\lambda_{c}^{+}(\phi, 0) \leqslant 2 n \mathcal{H} \mathcal{H}_{1-\delta_{n}}(\phi) \quad \forall \phi \in C_{+}^{0}(\partial U) .
$$

Proof. - For $\xi \in U-\{0\}$ fixed, let $\phi_{1}\left(x^{\prime}\right)=\left|x^{\prime}-\xi\right|^{2}, x^{\prime} \in \partial U$ (which is, of course, the restriction to $\partial U$ of a linear function).

The corresponding critical solution is the function

$$
u_{\infty}^{2 n}=|x-\xi|^{2},
$$

such that:

$$
\lambda_{c}^{+}\left(\phi_{1}, 0\right)=2 n=2 n \mathbb{H}_{1-n / 2}\left(\phi_{1}\right)
$$

Let $\phi_{2}$ be the trace on $\partial U$ of the harmonic function $1+\frac{1}{2} x_{1} x_{2}$. The corresponding critical solution is given by the formula

$$
u_{\infty}^{2 n}(x)=|x|^{2}+\frac{1}{2} x_{1} x_{2}
$$

such that $\lambda_{o}^{+}\left(\phi_{2}, 0\right)=2 n=2 n \mathcal{H}_{1}\left(\phi_{2}\right)$, where in the last step the mean value theorem for harmonic functions was used. The claim (ii) is an immediate consequence of (i) and of the following monotonicity property of the mean value: $\mathcal{M}_{t_{1}}(\phi)<$ $<\mathscr{N}_{t_{2}}(\phi)$ if $t_{1}<t_{2}$ and $\phi$ is nonconstant on $\partial B_{1}$.

Consider now the one dimensional ease $U=(-1,1)$. 
Proposition 1.4.3. - (i) Under assumption (1.4.4), one has:

$$
2 \mathcal{M}_{\frac{1}{2}}\left(\phi_{\infty}\right)+2 e^{-1} \mathcal{M}_{0}\left(g_{\infty}\right) \leqslant \lambda_{c}^{+}\left(\phi_{\infty}, g_{\infty}\right) \leqslant 2 \mathcal{M}_{1}\left(\phi_{\infty}\right)+\int_{-1}^{1}(1-|y|) g_{\infty}(y) d y .
$$

(ii) Under assumptions (1.4.4), (1.4.5), one has:

$$
\lambda_{0}^{+}\left(\phi_{\infty}^{\infty}, 0\right)=2 M_{\frac{1}{2}}\left(\phi_{\infty}\right) .
$$

Proof. - A direct computation shows that:

$$
\min _{|x|<1}\left(\left(1-x^{2}\right)^{-1} \sum_{\left|x^{\prime}\right|=1}\left(1+x^{\prime} x\right) \phi_{\infty}\left(x^{\prime}\right)\right)=2 \mathcal{M}_{1 / 2}\left(\phi_{\infty}\right) .
$$

This proves (1.4.9). The second inequality in (1.4.8) is obtained similarly as the upper bound for $\lambda_{c}^{+}$in (1.4.2). In order to prove the first inequality in (1.4.8), one uses (1.4.10) and Hölder's inequality:

$$
\begin{aligned}
& \lambda_{\mathrm{c}}^{+}\left(\phi_{\infty}, g_{\infty}\right)=\min _{|x|<1} 2\left(1-x^{2}\right)^{-1}\left(\frac{1}{2} \sum_{\left|x^{\prime}\right|=1}\left(1+x^{\prime} x\right) \phi_{\infty}\left(x^{\prime}\right)+\int_{-1}^{1} G(x, y) g_{\infty}(y) d y\right) \geqslant \\
& \geqslant \min _{|x|<1}\left(\left(1-x^{2}\right)^{-1} \sum_{\left|x^{\prime}\right|=1}\left(1+x^{\prime} x\right) \phi_{\infty}\left(x^{\prime}\right)\right)+\min _{|x|<1} 2\left(1-x^{2}\right)^{-1} \int_{-1}^{1} G(x, y) g_{\infty}(y) d y \geqslant \\
&>2 \mathcal{H}_{1 / 2}\left(\phi_{\infty}\right)+\varrho_{t} \mathcal{M}_{t}\left(g_{\infty}\right), \quad \forall t>0,
\end{aligned}
$$

with $\varrho_{t}$ the same as in Proposition 1.4.1. A computation shows that

$$
\varrho_{t}=2 \cdot\left(1+(t+1)^{-1} t\right)^{-t^{-1}(t+1)},
$$

so that $\lim _{t \downarrow 0} \varrho_{t}=2 e^{-1}$.

As an extension of (1.4.9) to the multidimensional case, one can mention the following fact: if $\phi_{\infty}\left(x^{\prime}\right)$ can be extended as a linear function on the ball, then $\lambda_{c}^{+}\left(\phi_{\infty}, 0\right)=\mathcal{M}_{1-n / 2}\left(\phi_{\infty}\right)$ (see (1.4.7)). Thus, an explicit formula can be given for $\lambda_{c}^{+}\left(\phi_{\infty}, 0\right)$ if $\phi_{\infty}$ can be extended as a linear function. It seems to be impossible to find such a formula for $\lambda_{c}^{+}\left(\phi_{\infty}, 0\right)$ for $n>1$ and $\phi_{\infty} \in C_{+}^{0}(\partial U)$ (see Remark 2 in [9]).

\subsection{The critical set in the case of the Laplacian.}

It will be assumed that

$$
A_{\infty}\left(x, \frac{\partial}{\partial x}\right)=-\Delta, \quad f_{\infty}=1
$$




\section{Denote}

$$
E_{c}\left(\phi_{\infty}, g_{\infty}\right) \stackrel{\text { def }}{=} E_{0}\left(v_{\infty}^{\lambda_{c}^{+}\left(\phi_{\infty}, g_{\infty}\right)}\right) \quad \forall\left(\phi_{\infty}, g_{\infty}\right) \in O_{+}^{0}(\partial U) \times C^{\alpha}(U)
$$

where $v_{\infty}^{\lambda}$ is the solution of (1.3.1). (If $\lambda_{c}^{+} \geqslant 0$, then the proof of Theorem 1.3.1 yields: $\left.\dot{E}_{e}\left(\phi_{\infty}, g_{\infty}\right)=E_{0}\left(u_{\infty}^{\lambda+\left(\phi_{\infty}, g_{\infty}\right)}\right)\right)$.

THEOREM 1.5.1. - Let $\left(\phi_{\infty}, g_{\infty}\right) \in{O^{0}}_{+}(\partial U) \times{O^{\alpha}}^{\alpha}(U)$ with $\alpha \in(0,1]$. Then for $\forall \xi \in$ $\in E_{e}\left(\phi_{\infty}, g_{\infty}\right)$, there exists $\psi_{\xi} \in C_{+}^{0}(\partial U)$ such that:

$$
E_{c}\left(\phi_{\infty}+\delta \psi_{\xi}, g_{\infty}\right)=\{\xi\}, \quad \forall \delta>0
$$

and, moreover, for the solution $v_{\delta}^{\lambda_{c}^{+}}$of (1.3.1) corresponding to the data $\phi_{\infty}+\delta \psi_{\xi}, g_{\infty}$, the matrix of second derivatives $D_{x}^{2} v_{\delta}(\xi)$ is positive definite, $\forall \delta>0$.

Proof. - Define the function $\psi_{\xi} \in C_{+}^{0}(\partial U)$ as follows:

$$
\psi_{\xi}\left(x^{\prime}\right)=\left|x^{\prime}-\xi\right|^{2}, \quad \forall x^{\prime} \in \partial U, \xi \in E_{c}\left(\phi_{\infty}, g_{\infty}\right)
$$

Denote by $w^{\lambda}$ the solution of $\mathfrak{H}_{\infty}^{\lambda}$ with $g_{\infty} \equiv 0$ and the boundary condition $\pi_{0} w^{\lambda}=\psi_{\xi}$. For $\lambda=\lambda_{c}^{+}\left(\psi_{\xi}, 0\right)$, one finds easily the corresponding critical solution:

$$
w_{c}^{\lambda_{c}^{+}(x)}=|x-\xi|^{2}=\Psi_{\xi}(x)-2 n v(x)
$$

where $\Psi_{\xi}$ is the harmonic function in $U$, such that $\pi_{0} \Psi_{\xi}=\psi_{\xi}$ and $v$ is defined by (1.4.1). Hence,

$$
\lambda_{c}^{+}\left(\psi_{\xi}\right)=\Delta w_{c}^{\lambda_{c}^{+}}(x)=2 n .
$$

Further, since $w^{\lambda_{c}^{+}}(x)>0, \forall x \in \bar{U} \backslash\{\xi\}$, one has:

$$
\Lambda_{p_{\xi}, 0}^{+}(x) \stackrel{\text { det }}{=}(v(x))^{-1} \Psi_{\xi}(x)>2 n=\Lambda_{\psi_{\xi}, 0}^{+}(\xi)=\min _{\nu \in \bar{U}} \Lambda_{\psi_{\xi}, 0}^{+}(y)
$$

Let $u_{\infty}^{0}(x)$ denote the solution of the problem $\mathfrak{A}^{\lambda}$ for $\lambda=0$. Using (1.5.5) and the fact that $\xi$ is a global minimum of $\Lambda_{\phi_{\infty}}^{+}$, $\infty_{\infty}$, one gets:

$$
\begin{aligned}
\Lambda_{\phi_{\infty}+\delta \psi_{\xi}, \nu_{\infty}}^{+}(x) \stackrel{\text { def }}{=}(v(x))^{-1}\left(u_{\infty}^{0}(x)+\delta \Psi_{\xi}(x)\right)>(v(x))^{-1} u_{\infty}^{0}(x)+\delta(v(\xi))^{-1} \Psi_{\xi}(\xi) \geqslant \\
\quad \geqslant(v(\xi))^{-1}\left(u_{\infty}^{0}(\xi)+\delta \Psi_{\xi}(\xi)\right)=\Lambda_{\phi_{\infty}+\delta \psi_{\xi}, g_{\infty}}(\xi), \quad \forall x \in \vec{U} \backslash\{\xi\} .
\end{aligned}
$$

Therefore, $\xi \in U$ is the only point where the function $\Lambda_{\phi_{\infty}+\delta w_{\xi}, o_{\infty}}(x)$ attains its 
minimum. As a consequence, the function

$$
v_{1}(x)=u_{\infty}^{0}(x)-\lambda_{c}^{+}\left(\phi_{\infty}+\delta \psi_{\xi}, g_{\infty}\right) v(x)
$$

is such that $E_{0}\left(v_{1}\right)=\{\xi\}$.

Now the second elaim of Theorem 1.5.1 will be proved. A straightforward computation using the relations

$$
v_{\delta}^{\lambda_{c}}(\xi)=0, \quad \nabla_{x} v_{\delta}^{\lambda_{c}}(\xi)=0
$$

yields the formula:

$$
D^{2} \Lambda_{\phi_{\infty}+\delta \psi_{\xi}, \theta_{\infty}}^{+}(\xi)=(v(\xi))^{-1} D^{2} v_{\delta}^{\lambda_{c}}(\xi), \quad \forall \xi \in E_{0}\left(u_{\delta}^{\lambda_{c}}\right)
$$

Hence,

$$
\begin{aligned}
D^{2} v_{\delta}^{\lambda_{o}}(\xi)=v(\xi) D^{2} \Lambda_{\phi_{\infty}+\delta \psi_{\xi}, \sigma_{\infty}}^{+}(\xi)=v(\xi)\left(D^{2} \Lambda_{\phi_{\infty}, o_{\infty}}^{+}(\xi)+\delta D^{2} \Lambda_{\psi_{\xi}, 0}^{+}(\xi)\right)= \\
=v(\xi) D^{2} \Lambda_{\phi_{\infty}, g_{\infty}}^{+}(\xi)+\left.\delta D_{x}^{2}\left(|x-\xi|^{2}\right)\right|_{x=\xi} \geqslant \delta 2 n I d,
\end{aligned}
$$

where $I d$ denotes the identity matrix.

Now we are going to prove that for $g_{\infty} \equiv 0$ and for a large class of boundary functions $\phi_{\infty}$, the set $E_{c}\left(\phi_{\infty}, 0\right)$ consists of only one point.

Definition 1.5.2. - For $\alpha \in(0,1], O_{+}^{\alpha}$ is defined to be the set of the pairs $\left(\phi_{\infty}, g_{\infty}\right) \in C_{+}^{0}(\partial U) \times C^{\alpha}(\bar{U})$, such that the critical set $E_{e}\left(\phi_{\infty}, g_{\infty}\right)$ contains only one point $\xi$ and such that, for the solution $v_{\infty}^{\lambda_{c}}\left(\phi_{\infty}, g_{\infty}\right)$ of (1.3.1), the Hessian $D^{2} v_{\infty}^{\lambda_{c}}(\xi)$ is positive definite at $\xi$.

As a consequence of Theorem 1.5.1, the set $O_{+}^{\alpha}$ is dense in $C_{+}^{0}(\partial U) \times C^{\alpha}(\bar{U})$.

Theorem 1.5.3. $-O_{+}^{\alpha}$ is open in $C_{+}^{0}(\partial U) \times C^{\alpha}(\vec{U})$.

Proof. - Let $\left(\phi_{\infty}, g_{\infty}\right) \in O_{+}^{\alpha}$ and $\{\xi\}=E_{c}\left(\phi_{\infty}, g_{\infty}\right)$.

With $\Lambda_{\phi_{\infty}, a_{\infty}}^{+}$defined by (1.3.3), one gets as in (1.5.6):

$$
D_{x}^{2} \Lambda_{\phi_{\infty}, g_{\infty}}^{+}(\xi)=(v(\xi))^{-1} D_{x}^{2} v_{\infty}^{\lambda_{c}}(\xi) \geqslant 2 \gamma I d
$$

where $\gamma>0$. Thus, there exists a constant $\delta_{1}>0$ such that

$$
D_{x}^{2} \Lambda_{\phi_{\infty}, g_{\infty}}^{+}(x) \geqslant \gamma I d, \quad \forall x \in B_{\delta_{1}}=\left\{x \in U:|x-\xi|<\delta_{1}\right\} .
$$

Since $\xi$ is the only point in the eritical set and therefore the only global minimum of $\Lambda_{\phi_{\infty}, g_{\infty}}^{+}(x)$, one can choose a constant $\delta_{2}>0$ such that

$$
\Lambda_{\phi_{\infty}, g_{\infty}}^{+}(x) \geqslant \Lambda_{\phi_{\infty}, g_{\infty}}^{+}(\xi)+\delta_{2}, \quad \forall x \in \bar{U} \backslash B_{\delta_{1}} .
$$


The inequalities (1.5.9), (1.5.10) will be used in order to show that there exists a constant $\varrho>0$ such that for any $\left(\psi_{\infty}, h_{\infty}\right) \in C_{+}^{0}(\partial U) \times C^{\alpha}(\bar{U})$, satisfying the condition

$$
\left[\psi_{\infty}-\phi_{\infty}\right]_{\sigma^{0}(\hat{\partial U})}+\left[h_{\infty}-g_{\infty}\right]_{C^{\alpha}(\bar{U})} \leqslant \varrho,
$$

the function $A_{\psi_{\infty}, h_{\infty}}^{+}$has still only one global minimum. Indeed, one has as a consequence of (1.3.3)

$$
\lambda_{c}^{+}\left(\psi_{\infty}, h_{\infty}\right)=\min _{x \in U} \Lambda_{\psi_{\infty}, h_{\infty}}^{+}(x) \leqslant \Lambda_{\psi_{\infty}, h_{\infty}}^{+}(\xi) \leqslant \Lambda_{\phi_{\infty}, g_{\infty}}^{+}(\xi)+\varrho\left(1+(v(\xi))^{-1}\right)
$$

Since

$$
A_{\psi_{\infty}, h_{\infty}}^{+}(x) \geqslant(v(x))^{-1}\left(\min \psi_{\infty}+v(x) \min h_{\infty}\right) \geqslant(v(x))^{-1}\left(\min \phi_{\infty}-\varrho\right)+g_{\infty}-\varrho,
$$

and since the function $v(x)$ vanishes on the boundary $\partial U$, one ean choose $\delta_{3}>0$ such that for $\varrho$ sufficiently small, all global minima of $\Lambda_{\psi_{\infty}, h_{\infty}}^{+}$are contained in the set

$$
U_{\delta_{3}}=\left\{x \in U: \operatorname{dist}(x, \partial U) \geqslant \delta_{3}\right\} \text {. }
$$

Indeed, the definition of $\Lambda_{\psi, h}^{+}$implies:

$$
\Lambda_{\psi_{\infty}, h_{\infty}}^{+}(x) \geqslant \Lambda_{\phi_{\infty}, g_{\infty}}^{+}(x)-\varrho\left(1+(v(x))^{-1}\right) .
$$

Further, (1.5.10), (1.5.11) yield the following inequalities:

$$
\begin{aligned}
& \Lambda_{\psi_{\infty}, h_{\infty}}^{+}(x) \geqslant \Lambda_{\phi_{\infty}, \sigma_{\infty}}^{+}(x)-\varrho\left(1+v(x)^{-1}\right) \geqslant \Lambda_{\phi_{\infty}, \sigma_{\infty}}^{+}(\xi)+\delta_{2}-\varrho\left(1+(v(x))^{-1}\right) \geqslant \\
& \quad \geqslant \Lambda_{\psi_{\infty}, h_{\infty}}^{+}(\xi)+\delta_{2}-\varrho\left(2+(v(x))^{-1}+(v(\xi))^{-1}\right) \geqslant \min \Lambda_{\psi_{\infty}, h_{\infty}}^{+}+\frac{\delta_{2}}{2}, \quad \forall x \in U_{\delta_{3}} \backslash B_{\delta_{1}},
\end{aligned}
$$

provided that $\varrho$ is sufficiently small. Therefore, for such $\varrho$, all global minima of $A_{\psi_{\infty}, h_{\infty}}^{+}$are contained in the set $B_{\delta_{1}}$. The following estimate, however, shows that the functions $A_{w_{\infty}}^{+}, h_{\infty}$ are strictly convex in $B_{\delta_{1}}$ for $\varrho$ sufficiently small. Using the interior Schander estimate and (1.5.9), one finds for $x \in B_{\delta_{1}}$ :

$$
\left\{\begin{array}{l}
D_{x}^{2} \Lambda_{y_{\infty}, h_{\infty}}^{+}(x)=D_{x}^{2} \Lambda_{\phi_{\infty}, o_{\infty}}^{+}(x)+D_{x}^{2}(v(x))^{-1} \\
\cdot\left(\int_{\partial U} E\left(x, y^{\prime}\right)\left(\psi_{\infty}\left(y^{\prime}\right)-\phi_{\infty}\left(y^{\prime}\right)\right) d \sigma_{y^{\prime}}+\int_{U} G(x, y)\left(h_{\infty}(y)-g_{\infty}(y)\right) d y\right), \\
D_{x}^{2} \Lambda_{\psi_{\infty}, h_{\infty}}^{+}(x) \geqslant\left(\gamma-C_{\varrho}\right) I d, \quad \forall x \in B_{\delta_{1}},
\end{array}\right.
$$

where $C>0$ is some constant. 
Hence, $A_{\psi_{\infty}, h_{\infty}}^{+}$has for $\varrho \ll 1$ only one global minimum and the set $E_{c}\left(\psi_{\infty}, h_{\infty}\right)$ contains only one point $\eta=\eta\left(\psi_{\infty}, h_{\infty}\right) \in B_{\delta_{1}}$. (1.5.8) and (1.5.12) yield that the matrix of second derivatives of the corresponding critical solution at the point $\eta$ is positive definite.

The next result follows immediately from the Theorems 1.5.1, 1.5.3:

Corollart 1.5.4. - The complement of $O_{+}^{\alpha}$ in $O_{+}^{0}(\partial U) \times C^{\alpha}(\bar{U})$ is nowhere dense.

THEoreMr 1.5.5. - Let $\left(\phi_{\infty}^{0}, g_{\infty}^{0}\right) \in O_{+}^{\alpha}$. Then the functional

$$
C_{+}^{0}(\partial U) \times C^{\alpha}(\bar{U}) \ni\left(\phi_{\infty}, g_{\infty}\right) \rightarrow \lambda_{c}^{+}\left(\phi_{\infty}, g_{\infty}\right) \in \boldsymbol{R}
$$

is Fréchet-differentiable at $\left(\phi_{\infty}^{0}, g_{\infty}^{0}\right)$ and its first variation in the direction $\left(\psi_{\infty}, g_{\infty}\right)$ is given by the formula:

$$
\delta \lambda_{c}^{+}\left(\phi_{\infty}^{0}, g_{\infty}^{0}\right) \circ\left(\psi_{\infty}, h_{\infty}\right)=(v(\xi))^{-1}\left(\int_{\partial U} E\left(\xi, y^{\prime}\right) \psi_{\infty}\left(y^{\prime}\right) d \sigma_{y^{\prime}}+\int_{U} G(\xi, y) h_{\infty}(y) d y\right) .
$$

Here $\{\xi\}=E_{c}\left(\phi_{\infty}^{0}, g_{\infty}^{0}\right), G$ is Green's function of the Dirichlet problem with zero boundary condition for the Poisson equation in $U$, and the functions $E$ and $v$ are defined by $E\left(x, y^{\prime}\right)=\pi_{0}\left(\partial / \partial N_{y^{\prime}}\right) G\left(x, y^{\prime}\right)$ and (1.4.1), respectively.

Proof. - First, one has to show that the function

$$
s \rightarrow \lambda_{c}^{+}\left(\phi_{\infty}^{0}+s \psi_{\infty}, g_{\infty}^{0}+s h_{\infty}\right)
$$

is differentiable at $s=0, \forall\left(\psi_{\infty}, h_{\infty}\right) \in C^{0}(\partial U) \times C^{\alpha}(\vec{U})$.

Since $O_{+}^{\alpha}$ is open in $O^{0}(\partial U) \times O^{\alpha}(\vec{U})$, one has the following formula:

$$
\lambda_{c}^{+}\left(\phi_{\infty}^{0}+s \psi_{\infty}, g_{\infty}^{0}+s h_{\infty}\right)=\Lambda_{\phi_{\infty}^{0}+s \psi_{\infty}, g_{\infty}^{0}+s h_{\infty}}^{+}(\xi(s))
$$

where $A_{\psi, h}^{+}$is defined by (1.3.3) and $\xi(s)$ is well defined by

$$
\{\xi(s)\}=E_{c}\left(\phi_{\infty}^{0}+s \psi_{\infty}, g_{\infty}^{0}+s h_{\infty}\right)
$$

for $|s|$ sufficiently small.

Besides, one has for $\xi(s)$ the following equations:

$$
\nabla_{x} \Lambda_{\phi_{\infty}^{0}+s \psi_{\infty}, \theta_{\infty}^{\circ}+s \hbar_{\infty}}^{+}(\xi(s))=0
$$

Since, as a consequence of (1.5.8),

$$
D_{x}^{2} \Lambda_{\phi_{\infty}^{0}, g_{\infty}^{0}}^{+}(\xi(0))=v(\xi(0))^{-1} D_{x}^{2} v_{\infty}^{\lambda_{c}}(\xi(0)) \geqslant \gamma I d, \quad \gamma>0,
$$


the implicit function theorem yields: the function $s \rightarrow \xi(s) \in U$ is differentiable for $|s|$ sufficiently small. As a consequence of (1.5.16), the regularity of the funetion $\Lambda_{\phi, h}^{+}(x), x \in U$ and the differentiability of $\xi(s)$, one gets the conclusion that the function (1.5.15) is differentiable at $s=0$.

A straightforward computation using the relations:

$$
v_{\infty}^{\lambda_{\infty}^{+}\left(\phi_{\infty}^{0}, g_{\infty}^{0}\right)}(\xi(0))=0, \quad \nabla_{x} v_{\infty}^{\lambda_{\infty}^{+}\left(\phi_{\infty}^{0}, g_{\infty}^{0}\right)}(\xi(0))=0
$$

then yields the formula (1.5.14).

Theorem 1.5.6. - Let $U \subset \boldsymbol{R}^{2}$ be a bounded, simply connected domain and let $\phi_{\infty} \in C_{+}^{0}(\partial U), g_{\infty} \equiv 0$. If the coefficients $a_{k}(x)$ of the differential operator

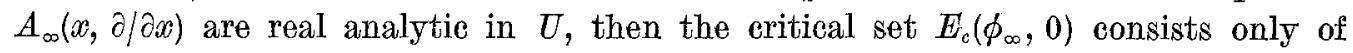
a finite number of points.

THEOREM 1.5.7. - If $U \subset \subset \boldsymbol{R}^{2}$ is a bounded domain (not necessarily simply connected) and if the coefficients of the differential operator $A_{\infty}$ are real analytic, then $E_{c}\left(\phi_{\infty}, 0\right)$ is the collection of finitely many isolated points and a finite number of closed analytic curves.

The proof of Theorems 1.5.6, 1.5.7 stated above is similar to the proof of Theorem 5 and Corollary 2 in [9].

It should be mentioned that even if $U$ is the unit disk in $\boldsymbol{R}^{2}$, there exist functions $\phi_{\infty} \in C_{+}^{0}(\partial U)$, such that the set $E_{c}\left(\phi_{\infty}, 0\right)$ contains more than one point. Moreover, in this case the set $E_{0}\left(u_{\infty}^{\lambda}\right)$ is not necessarily connected (see [9]).

\subsection{The asymptotic behaviour of the solution to $\mathfrak{A}_{\infty}^{\lambda}$ when $\lambda \rightarrow+\infty$.}

In this section, an asymptotic formula for the solution $u_{\infty}^{\lambda}$ of $\mathfrak{Q}_{\infty}^{\lambda}$ is indicated and an error estimate in the maximum norm is stated. One uses super- and subsolutions of special type and the maximum principle for establishing this result (see [9], Theorem 7 for the case $A_{\infty}=-4$ ).

First, consider the case

$$
f_{-\infty}<0<f_{\infty}
$$

For simplicity, it will be assumed from now on, that

$$
\phi_{\infty} \in C_{+}^{0} \cap C^{2}(\partial U)
$$

For $x \in U$ in a sufficiently small neighbourhood of $\partial U$, let $x^{\prime} \in \partial U$ and $\varrho$ be defined by

$$
\left|x-x^{\prime}\right|=\operatorname{dist}(x, \partial U)=\min _{y^{\prime} \in \partial U}\left|x-y^{\prime}\right|=\varrho
$$


Using the coordinates $x^{\prime}, \varrho$, one can rewrite the operator $A_{\infty}$ as follows:

$$
A_{\infty}\left(x, \frac{\partial}{\partial x}\right)=-a\left(x^{\prime}, \varrho\right) \frac{\partial^{2}}{\partial \varrho^{2}}+B
$$

Here $a\left(x^{\prime}, \varrho\right)$ is a smooth positive function and the differential operator $B$ has orders 2 and 1 in $\partial / \partial x^{\prime}$ and $\partial / \partial \varrho$, respectively. Using the notation

$$
a\left(x^{\prime}\right)=a\left(x^{\prime}, 0\right)=\sum_{1 \leqslant k, j \leqslant n} a_{k j}^{\infty}\left(x^{\prime}\right) N_{k}\left(x^{\prime}\right) N_{j}\left(x^{\prime}\right)
$$

and the normalized distance $\varrho_{1}$,

$$
\varrho_{1}=\left(\lambda f_{\infty} \cdot\left(2 a\left(x^{\prime}\right)\right)^{-1}\right)^{1 / 2} \varrho,
$$

one has:

Treorem 1.6.1. - Under the assumptions (1.6.1), (1.6.2), the function

$$
w_{\infty}^{\lambda}(x)=\left(\left(\phi_{\infty}\left(x^{\prime}\right)\right)^{1 / 2}-\varrho_{1}\right)_{+}^{2},
$$

where $s_{+}=\max (s, 0)$, is an asymptotic solution of $\mathfrak{A}_{\infty}^{\lambda}$ such that

$$
\left\|u_{\infty}^{\lambda}-w_{\infty}^{\lambda}\right\|_{C^{\circ}(\bar{V})} \leqslant C \lambda^{-1 / 2}
$$

where the constant $C$ does not depend on $\lambda$. Moreover, for the free boundary $\partial E_{0}\left(u_{\infty}^{\lambda}\right)$ holds:

$$
\partial E_{0}\left(u_{\infty}^{\lambda}\right) \subset\left\{x \in U:\left|\left(2 \phi_{\infty}\left(x^{\prime}\right) a\left(x^{\prime}\right)\left(\lambda f_{\infty}\right)^{-1}\right)^{1 / 2}-\varrho\right| \leqslant C_{1} \lambda^{-1}\right\}
$$

where the constant $C_{1}$ does not depend on $\lambda$.

Assuming again (1.6.2), we consider now the case $0=f_{-\infty}<f_{\infty}$.

If $g_{\infty}(x) \geqslant 0, \forall x \in \bar{U}$, then the function (1.6.3) is still an asymptotic solution of $\mathfrak{A}_{\infty}^{\lambda}$ when $\lambda \rightarrow \infty$. Using super- and subsolutions, one shows that (1.6.4), (1.6.5) are valid in this case, as well.

Ig $g_{\infty}(x)<0, \forall x \in \bar{U}, g_{\infty} \in C^{\alpha}(\bar{U})$, then it will turn out that for $\lambda \rightarrow \infty$, the solution $u^{\lambda}$ converges on any $U_{1} \subset \subset U$ to the solution $z(x)$ of the linear boundary value problem

$$
\begin{array}{ll}
A_{\infty}\left(x, \frac{\partial}{\partial x}\right) z(x)=g_{\infty}(x), & x \in U, \\
\pi_{0} z\left(x^{\prime}\right)=0, & x^{\prime} \in \partial U .
\end{array}
$$

In a neighbourhood of $\partial U$ the solution $u_{\infty}^{\lambda}$ has again a boundary layer behaviour as $\lambda \rightarrow \infty$. 
Let $\beta\left(x^{\prime}\right)=\pi_{0}(\partial z / \partial N)\left(x^{\prime}\right), x^{\prime} \in \partial U$, and define $\xi\left(x^{\prime}\right)$ to be the positive solution of the quadratic equation

$$
\beta\left(x^{\prime}\right) \xi\left(x^{\prime}\right)+\left(\lambda f_{\infty} / 2 a\left(x^{\prime}\right)\right) \xi\left(x^{\prime}\right)^{2}=\phi_{\infty}\left(x^{\prime}\right) .
$$

Let $U_{\xi}=\left\{x \in U: \varrho<\xi\left(x^{\prime}\right)\right\}$ and denote by $w_{\infty}^{\lambda}(x), x \in U \backslash U_{\xi}$, the solution of the following boundary value problem:

$$
\begin{cases}A_{\infty}\left(x, \frac{\partial}{\partial x}\right) w_{\infty}^{\lambda}(x)=g_{\infty}(x), & x \in U \backslash U_{\xi}, \\ w_{\infty}^{\lambda}\left(x^{\prime}\right)=0, & x^{\prime} \in \partial U_{\xi} \backslash \partial U .\end{cases}
$$

On the set $U_{\xi}$, we define $w_{\infty}^{2}$ as follows:

$$
w_{\infty}^{\lambda}\left(\varrho, x^{\prime}\right)=\beta_{\lambda}\left(x^{\prime}\right)\left(\xi\left(x^{\prime}\right)-\varrho\right)+\left(\lambda f_{\infty} / 2 a\left(x^{\prime}\right)\right)\left(\xi\left(x^{\prime}\right)-\varrho\right)^{2}, \quad 0<\varrho<\xi\left(x^{\prime}\right)
$$

where $\beta_{\lambda}\left(x^{\prime}\right)$ denotes the restriction of the normal derivative to $\partial U_{\xi} \backslash \partial U$ of the solution of (1.6.6).

The function $w_{\infty}^{\lambda}$ defined above, is a formal asymptotic solution of $\mathfrak{H}_{\infty}^{\lambda}$ when $\lambda \rightarrow \infty$ :

$$
\begin{cases}A_{\infty}\left(x, \frac{\partial}{\partial x}\right) w_{\infty}^{\lambda}+(\lambda+O(\sqrt{\lambda})) f_{0}\left(w_{\infty}^{\lambda}\right)+g_{\infty}(x) \chi_{0}\left(w_{\infty}^{\lambda}\right)=g_{\infty}(x), & x \in U, \\ 0 \leqslant g_{\infty}(x) \leqslant \lambda f_{\infty}, & x \in \operatorname{int} E_{0}\left(w_{\infty}^{\lambda}\right), \\ \pi_{0} w_{\infty}^{\lambda}\left(x^{\prime}\right)=\phi_{\infty}\left(x^{\prime}\right)+O\left(\lambda^{-1 / 2}\right), & x^{\prime} \in \partial U .\end{cases}
$$

Note that the boundary condition is satisfied asymptotically because $\xi\left(x^{\prime}\right)=O\left(\lambda^{-1 / 2}\right)$ when $\lambda \rightarrow \infty$ holds uniformly w.r.t. $x^{\prime} \in \partial U$ and because the Schauder estimate implies that $\left[\beta-\beta_{\lambda}\right]_{C^{\circ}(\partial U)}=O\left(\lambda^{-1 / 2}\right)$ when $\lambda \rightarrow \infty$.

Using a maximum principle argument, one finds that $w_{\infty}^{\lambda}$ is an asymptotic solution of $\mathfrak{H}_{\infty}^{\lambda}$ when $\lambda \rightarrow \infty$.

Note that in the case $f_{-\infty}=0<f_{\infty}$, independently on the sign of $g_{\infty}(x)$, $u^{\lambda}$ converges to the solution $u$ of the following problem $\mathfrak{A}_{\infty}$ :

$$
\begin{cases}A_{\infty}\left(x, \frac{\partial}{\partial x}\right) u=g_{\infty}(x)\left(1-\chi_{0}(u)\right), & x \in U, \\ 0 \leqslant g_{\infty}(x), & x \in \operatorname{int}\left(E_{0}(u)\right), \\ u(x) \leqslant 0, & x \in U, \\ \pi_{0} u\left(x^{\prime}\right)=0, & x^{\prime} \in \partial U .\end{cases}
$$

In general, $\mathfrak{A}_{\infty}$ is, of course, a free boundary problem. 
It can be reformulated as a minimization problem as follows:

$$
\min _{\substack{u \in \mathrm{O}_{1}(U) \\ u \leqslant 0}} \int_{U}\left(\frac{1}{2} \sum a_{k_{j}}(x) u_{x_{j}} u_{x_{k}}-g_{\infty}(x) u(x)\right) d x .
$$

Finally, consider the case $f_{-\infty}<0=f_{\infty}$.

For $\lambda \geqslant \max _{x \in U}\left(f_{-\infty}^{-1} g_{\infty}(x)\right)$, the function $u_{\infty}^{\lambda}$ does not depend upon $\lambda$ and coincides with the solution $u$ of the following problem $\mathfrak{A}_{\infty}$ :

$$
\begin{cases}A_{\infty} u(x)=g_{\infty}(x)\left(1-\chi_{0}(u)\right), & x \in U, \\ g_{\infty}(x) \leqslant 0, & x \in \operatorname{int}\left(E_{0}(u)\right) \\ u(x) \geqslant 0, & x \in U, \\ \pi_{0} u\left(x^{\prime}\right)=\phi_{\infty}\left(x^{\prime}\right), & x^{\prime} \in \partial U\end{cases}
$$

The equivalent formulation as a minimization problem reads as follows:

$$
\min _{\substack{u \in H_{1}(U) \\ \pi_{0} u=\phi_{\infty} \\ u \geqslant 0}} \int_{U}\left(\frac{1}{2} \sum a_{k j}(x) u_{x_{j}} u_{x_{k}}-g_{\infty}(x) u(x)\right) \mathrm{d} x .
$$

REMARK 1.6.2. - One can consider a problem with more general non-linearity:

$$
\left\{\begin{aligned}
-\Delta u_{\infty}^{\lambda}+\lambda q^{\prime}\left(u^{\lambda}\right) \chi_{+}\left(u_{\infty}^{\lambda}\right) & =0, & & x \in U, \\
\pi_{0} u_{\infty}^{\lambda}\left(x^{\prime}\right) & =\phi\left(x^{\prime}\right), & & x^{\prime} \in U
\end{aligned}\right.
$$

where $\phi\left(x^{\prime}\right)>0, \forall x^{\prime} \in \partial U, \lambda \gg \phi$ and $q^{\prime}(s)$ is monotonically increasing on the interval $\left[0, \phi_{M}\right]$ with $\phi_{M}=\max _{x^{\prime} \in \partial U} \phi\left(x^{\prime}\right)$.

Then the corresponding asymptotic solution $w_{\infty}^{\lambda}\left(x^{\prime}, Q\right)$ is defined by the formula:

$$
\int_{\substack{w_{\infty}^{2}\left(x^{\prime}, \varrho\right) \\ \phi\left(x^{\prime}\right)}} \frac{d s}{\sqrt{2 q(s)}}=\sqrt{\lambda} \varrho,
$$

where $q(s)$ is the primitive of $q^{\prime}(s)$ normalized by the condition $q(0)=0$. Moreover for the free boundary $\partial E_{0}\left(u_{\infty}^{\lambda}\right)$ of $u_{\infty}^{\lambda}$, solution to (1.6.7), holds:

$$
\partial E_{0}(u) \subset\left\{x \in U:\left|\int_{0}^{\phi\left(x^{\prime}\right)} \frac{d s}{\sqrt{2 \varrho q(s)}}-\varrho\right| \leqslant C_{1} \lambda^{-1}\right\},
$$

where $C_{1}>0$ is some constant.

In case of a general second order elliptic operator the distance $\varrho$ in (1.6.8), (1.6.9) has to be replaced by the normalized distance $\varrho_{1}$ defined here above. 


\section{2. - Nonstationary problem.}

2.1. General properties of the operators considered.

Denote

$$
B\left(Q_{T}\right)=L^{2}\left(Q_{T}\right) \times H_{1}(U) \times H_{3 / 2,3 / 4}\left(\Gamma_{T}\right), \quad 0<T<\infty,
$$

where $H_{s, r}\left(\Gamma_{T}\right)$ is the Sobolev space of all functions $\phi\left(x^{\prime}, t\right)$ such that $D_{x^{\prime}}^{\alpha} D_{t}^{m} \phi \epsilon$ $\in L^{2}\left(\Gamma_{T}\right), \forall|\alpha| \leqslant s, m \leqslant r$ for $s \geqslant 0, r \geqslant 0$ integer; if $s$ and $r$ are not necessarily integer, then $H_{s, r}\left(\Gamma_{T}\right)$ is defined in a standard way by using the partition of unity and the Fourier transform. Denote by $\mathfrak{A}_{\varepsilon}^{\lambda}$ the operator associated with the initial-boundary value problem $(0.6)$ :

$$
\mathfrak{A}_{\varepsilon}^{\lambda}: H_{2,1}\left(Q_{T}\right) \rightarrow B\left(Q_{T}\right), \quad 0<T<\infty .
$$

Theorem 2.1.1. - For any given $\varepsilon>0$, the mapping (2.1.2) is a Lipschitz-continuous homeomorphism.

THEOREM 2.1.2. - If $(g, \psi, \phi) \in C^{0}(\bar{Q}) \times C^{2}(\bar{U}) \times C^{2,1}(T)$ and the compatibility condition $(0.7)$ is satisfied, then for $\forall \alpha \in[0,1)$ uniformly with respect to $\varepsilon \in\left(0, \varepsilon_{0}\right]$ holds:

$$
u_{\varepsilon}^{\lambda} \in C^{1, \alpha ;(1+\alpha) / 2}(\bar{Q}) \text {. }
$$

Theorem 2.1.3. - If $(g, \psi, \phi) \in C^{0}(\bar{Q}) \times C^{2}(\bar{U}) \times C^{2,1}(\Gamma)$ and $(0.7)$ is satisfied, then. the reduced problem $\mathfrak{A}^{\lambda}$ has a well-defined (distributional) solution $u^{\lambda} \in C^{1, \alpha ;(1+\alpha) / 2}(\bar{Q})$ $\forall \alpha \in[0,1)$.

Moreover, the set $\left\{u_{\varepsilon}^{\lambda}\right\}_{0<\varepsilon \leqslant \varepsilon_{0}} \subset C^{1, \alpha ;(1+\alpha) / 2}\left(Q_{T}\right)$, where $u_{\varepsilon}^{\lambda}$ is the solution of $\mathfrak{U}_{\varepsilon}^{\lambda}$, has for $\forall T<\infty$ as its only condensation point the solution $u^{\lambda}$ of $\mathfrak{P}^{\lambda}$ when $\varepsilon \downarrow 0$, so that

$$
\lim _{\delta \downarrow \downarrow 0}\left\|u_{\varepsilon}^{\lambda}-u^{\lambda}\right\|_{O^{1, \alpha ;}(1+\alpha) / 2\left(\bar{Q}_{T}\right)}=0 \quad \forall T<\infty, \forall \alpha \in[0,1) .
$$

The proof of the Theorems 2.1.1, 2.1.2 and 2.1.3 will be given in a coming authors' publication.

\subsection{Convergence as $t \rightarrow \infty$.}

For simplicity, we assume that

$$
A\left(x, t, \frac{\partial}{\partial x}\right) \equiv A_{\infty}\left(x, \frac{\partial}{\partial x}\right), \quad \phi\left(x^{\prime}, t\right) \equiv \phi_{\infty}\left(x^{\prime}\right), g(x, t) \equiv g_{\infty}(x) .
$$

Let $\mu>0$ be the least eigenvalue of $A_{\infty}$ in $U$ with Dirichlet boundary condition on $\partial U$. 
THEOREM 2.2.1. - The stationary solutions $u_{\varepsilon, \infty}^{\lambda}, u_{\infty}^{\lambda}$ of the problems $\mathfrak{A}_{\varepsilon}^{\lambda}$ and $\mathfrak{A} \lambda$, respectively, are asymptotically stable in $L^{2}(U)$ as $t \rightarrow \infty$, and, moreover, the following estimates hold:

$$
\begin{cases}{\left[u_{\varepsilon}^{\lambda}(\cdot, t)-u_{\varepsilon, \infty}^{\lambda}\right]_{L_{2}(U)} \leqslant \exp (-\mu t)\left[\psi-u_{\varepsilon, \infty}^{\lambda}\right]_{L_{2}(U)}} & \forall t \geqslant 0, \forall \varepsilon>0 \\ {\left[u^{\lambda}(\cdot, t)-u_{\infty}^{\lambda}\right]_{L_{2}(U)} \leqslant \exp (-\mu t)\left[\psi-u_{\infty}^{\lambda}\right]_{L_{2}(U)}} & \forall t \geqslant 0\end{cases}
$$

Proof. - The difference $w_{\varepsilon}^{\lambda}=u_{\varepsilon}^{\lambda}-u_{\varepsilon, \infty}^{\lambda}$ is the solution of the following problem:

$$
\begin{cases}\frac{\partial w_{\varepsilon}^{\lambda}}{\partial t}+A_{\infty}\left(x, \frac{\partial}{\partial x}\right) w_{\varepsilon}^{\lambda}+\lambda\left(f\left(\varepsilon^{-1} u_{\varepsilon}^{\lambda}\right)-f\left(\varepsilon^{-1} u_{\varepsilon, \infty}^{\lambda}\right)\right)=0, & (x, t) \in Q, \\ w_{\varepsilon}^{\lambda}(x, 0)=\psi(x)-u_{\varepsilon, \infty}^{\lambda}(x), & x \in \bar{U}, \\ \pi_{0} w_{\varepsilon}^{\lambda}\left(x^{\prime}, t\right)=0, & \left(x^{\prime}, t\right) \in \Gamma .\end{cases}
$$

Multiplying the differential equation by $w_{\varepsilon}^{2}$, integrating by parts and using the fact that $f$ is monotonically increasing, one gets the following inequality:

$$
\frac{1}{2} \frac{d}{d t}\left[w_{\varepsilon}^{\lambda}(\cdot, t)\right]_{L_{2}(U)}^{2}+\left(A_{\infty} w_{\varepsilon}^{\lambda}, w_{\varepsilon}^{\lambda}\right) \leqslant 0, \quad \forall t \geqslant 0 .
$$

Now the inequality

$$
\left(A_{\infty} w, w\right) \geqslant \mu[w]_{L_{\mathbf{s}}(U)}^{2} \quad \forall w \in \stackrel{\cap}{H}_{1}(U)
$$

and Gronwall's Lemma yield the first of the inequalities (2.2.2). The second inequality in (2.2.2) follows from the first one and from (2.1.3).

THEOREM 2.2.2. - The following estimates hold under the assumption (2.2.1):

$$
\begin{aligned}
& {\left[\left(u_{\varepsilon}^{\lambda}\right)_{t}(\cdot, t)\right]_{L_{2}(U)} \leqslant \exp (-\mu t)\left[A_{\infty} \psi+\lambda f\left(\varepsilon^{-1} \psi\right)-g_{\infty}\right]_{L_{2}(U)} \quad \forall t, \varepsilon>0,} \\
& \left(A_{\infty}\left(u_{\varepsilon}^{\lambda}-u_{\varepsilon, \infty}^{\lambda}\right), u_{\varepsilon}^{\lambda}-u_{\varepsilon, \infty}^{\lambda}\right)_{L_{2}(U)}^{1 / 2} \leqslant \\
& \quad \leqslant \exp (-\mu t)\left[A_{\infty} \psi+\lambda f\left(\varepsilon^{-1} \psi\right)-g_{\infty}\right]_{L_{3}(U)}^{1 / 2}\left[\psi-u_{\varepsilon, \infty}^{\lambda}\right]_{L_{2}(U)}^{1 / 2} \quad \forall t, \varepsilon>0 .
\end{aligned}
$$

Proof. - Denote $v_{\varepsilon}^{\lambda}=\left(u_{\varepsilon}^{\lambda}\right)_{t}$, so that $v_{\varepsilon}^{\lambda}$ is the solution of the following problem:

$$
\begin{cases}\frac{\partial v_{\varepsilon}^{\lambda}}{\partial t}+A_{\infty}\left(x, \frac{\partial}{\partial x}\right) v_{\varepsilon}^{\lambda}+\varepsilon^{-1} \lambda j^{\prime}\left(\varepsilon^{-1} u_{\varepsilon}^{\lambda}\right) v_{\varepsilon}^{\lambda}=0, & (x, t) \in Q, \\ v_{\varepsilon}^{\lambda}(x, 0)=g_{\infty}-A_{\infty} \psi-\lambda f\left(\varepsilon^{-1} \psi\right), & x \in \bar{U}, \\ \pi_{0} v_{\varepsilon}^{\lambda}\left(x^{\prime}, t\right)=0, & \left(x^{\prime}, t\right) \in \Gamma .\end{cases}
$$


Since $f^{\prime}(s) \geqslant 0$, one gets, using (2.2.7):

$$
\frac{1}{2} \frac{d}{d t}\left[v_{\varepsilon}^{\lambda}(\cdot, t)\right]_{L_{2}(U)}^{2}+\left(A_{\infty} v_{\varepsilon}^{\lambda}, v_{\varepsilon}^{\lambda}\right) \leqslant 0 \quad \forall t \geqslant 0
$$

Further, (2.2.4) and Gronwall's lemma yield (2.2.5). Using (2.2.3), (2.2.4) and (2.2.5), one obtains for $w_{\varepsilon}^{\lambda}=u_{\varepsilon}^{\lambda}-u_{e, \infty}^{\lambda}$ :

$$
\begin{aligned}
& \left(A_{\infty} w_{\varepsilon}^{\lambda}, w_{\varepsilon}^{\lambda}\right)_{L_{2}(J)}^{1 / 2} \leqslant\left[\left(u_{\varepsilon}^{\lambda}\right)_{t}(\cdot, t)\right]_{L_{2}(U)}^{1 / 2}\left[w_{\varepsilon}^{\lambda}\right]_{L_{\varepsilon}(U)}^{1 / 2} \leqslant \\
& \quad \leqslant \exp (-\mu t)\left[A_{\infty} \psi+\lambda f\left(\varepsilon^{-1} \psi\right)-g_{\infty}\right]_{L_{s}(U)}^{1 / 2}\left[\psi-u_{\varepsilon, \infty}^{\lambda}\right]_{L_{2}(U)}^{1 / 2} .
\end{aligned}
$$

\subsection{Convergence for $\varepsilon \downarrow 0$.}

For simplicity, it is assumed that

$$
g \equiv 0, \quad \psi \geqslant 0, \quad \phi \geqslant 0
$$

Proposition 2.3.1. - Under the assumptions (0.7), (2.3.1), the following estimates hold:

$$
\left\{\begin{array}{l}
\left\|u_{\varepsilon}^{\lambda}(x, t)-u^{\lambda}(x, t)\right\|_{L_{L_{2}\left([0, T], H_{1}(U)\right)} \leqslant C(T \varepsilon)^{1 / 2}} \quad \forall T>0 \\
\left\|u_{\varepsilon}^{\lambda}(x, t)-u^{\lambda}(x, t)\right\|_{L_{L_{2}\left([0, \infty], L_{2}(U)\right)} \leqslant C \varepsilon^{1 / 2}}
\end{array}\right.
$$

where the constant $C$ depends only upon $\lambda$, meas $U, f$, the ellipticity constant of $A$ and upon the first eigenvalue of $(-\Delta)$ in $U$ with Dirichlet boundary conditions on $\partial U$.

Proof. - The difference $v_{\varepsilon}^{\lambda}=u_{\varepsilon}^{\lambda}-u^{\lambda}$ is a solution of the following problem.

$$
\begin{cases}\left(v_{\varepsilon}^{\lambda}\right)_{t}-A v_{\varepsilon}^{\lambda}+\lambda\left(f\left(\frac{u_{\varepsilon}^{\lambda}}{\varepsilon}\right)-f_{0}\left(u^{\lambda}\right)\right)=0, & (x, t) \in Q, \\ v_{s}^{\lambda}(x, 0)=0, & x \in U, \\ \pi_{0} v_{\varepsilon}^{\lambda}\left(x^{\prime}, t\right)=0, & x^{\prime} \in \partial U, t \in \boldsymbol{R}_{+} .\end{cases}
$$

Multiplying the differential equation by $v_{\varepsilon}^{\lambda}$ and integrating by parts, one obtains

$$
\begin{aligned}
\frac{1}{2} \frac{d}{d t}\left[v_{\varepsilon}^{\lambda}\right]_{L_{2}}^{2}\left(U^{2}\right)^{*}+\sum_{k, j} \int_{U} a_{k_{j}}(x, t)\left(v_{\varepsilon}^{\lambda}\right)_{k}\left(v_{\varepsilon}^{\lambda}\right)_{j} d x+\lambda \int_{E_{0}\left(u^{\lambda}\right) \cup E_{-}\left(u^{2}\right)} f\left(\frac{u_{\varepsilon}^{\lambda}}{\varepsilon}\right) & v_{\varepsilon}^{\lambda} d x= \\
& =\lambda \int_{E_{+}\left(u^{\lambda}\right)}\left(f_{0}\left(u^{\lambda}\right)-f\left(\frac{u_{\varepsilon}^{\lambda}}{\varepsilon}\right)\right) v_{\varepsilon}^{\lambda} d x .
\end{aligned}
$$


As a consequence of the assumption (2.3.1) and of Proposition 2.4.1 below, $E_{-}\left(u^{\lambda}\right)=\emptyset$ and $v_{\varepsilon}^{\lambda} \geqslant 0$. Thus,

$$
\frac{1}{2}\left[v_{\varepsilon}^{\lambda}(\cdot, T)\right]_{L_{2}(U)}^{2}+\gamma \int_{Q_{T}}\left|\nabla_{x} v_{\varepsilon}^{\lambda}\right|^{2} d x d t \leqslant \lambda \int_{Q_{T}}\left(f_{\infty}-f\left(\frac{u_{\varepsilon}^{\lambda}}{\varepsilon}\right)\right) v_{\varepsilon}^{\lambda} d x d t
$$

where $y>0$ is the ellipticity constant of $A$.

Thus,

$$
\frac{1}{2} \frac{a}{d t}\left[v_{\varepsilon}^{\lambda}\right]_{L_{2}(U)}^{2}+\gamma \mu\left[v_{\varepsilon}^{\lambda}\right]_{L^{2}(U)}^{2} \leqslant \lambda \int_{E_{+}\left(u^{\lambda}\right)}\left(f_{0}\left(u^{\lambda}\right)-f\left(\frac{u_{\varepsilon}^{\lambda}}{\varepsilon}\right)\right) v_{\varepsilon}^{\lambda} d x
$$

where $\gamma>0$ is the ellipticity constant and $\mu>0$ is the least eigenvalue of $-\Delta$ with homogeneous Dirichlet boundary condition on $\partial U$.

The integral in the right hand side of the last inequality can be estimated as follows:

$$
\int_{U}\left(f_{\infty}-f\left(\frac{u_{\varepsilon}^{\lambda}}{\varepsilon}\right)\right) v_{\varepsilon}^{\lambda} d x \leqslant C \int_{U} \frac{\varepsilon}{\varepsilon+u_{\varepsilon}^{\lambda}}\left(u_{\varepsilon}^{\lambda}-u^{\lambda}\right) d x \leqslant C \varepsilon \int_{U} \frac{u_{\varepsilon}^{\lambda}}{\varepsilon+u_{\varepsilon}^{\lambda}} d x \leqslant C \varepsilon \text { meas } U
$$

Using (2.3.4), (2.3.5) and Gronwall's lemma, one gets the second of the inequalities (2.3.3).

Integrating (2.3.4) over $[0, T]$ one gets the first of the inequalities (2.3.2).

Similarly to section 1.2 , an improved estimate for the rate of convergence of $u_{\varepsilon}^{\lambda}$ to $u^{\lambda}$ can be obtained under the same assumptions upon the free boundary of the solution $u^{\lambda}$. However, the construction of asymptotic solutions in this case is somewhat more tedious than for the stationary problem.

\subsection{Nonnegative solutions.}

Proposition 2.4.1. - If $g \geqslant 0, \psi \geqslant 0, \phi \geqslant 0$, then $u_{\varepsilon}^{\lambda} \geqslant 0, u^{\lambda} \geqslant 0$.

Proof. - Assume that the set $E_{-}\left(u_{\varepsilon}^{\lambda}\right)$ is nonempty. For $\forall(x, t) \in E_{-}\left(u_{\varepsilon}^{\lambda}\right)$, one has:

$$
\left(u_{\varepsilon}^{\lambda}\right)_{t}+A u_{\varepsilon}^{\lambda}=g(x, t)-\lambda f\left(\varepsilon^{-1} u_{\varepsilon}^{\lambda}\right) \geqslant 0
$$

Moreover, $u_{\varepsilon}^{\lambda}(x, t)=0$ for $\forall(x, t) \in \partial W_{-}\left(u_{\varepsilon}^{\lambda}\right)$. The maximum principle yields $u_{\varepsilon}^{\lambda} \geqslant 0$ in $E_{-}\left(u_{\varepsilon}^{\lambda}\right)$. Thus one obtains a contradiction. The nonnegativity of $u^{\lambda}$ is proved similarly.

Let $v_{\varepsilon}^{\lambda}$ be the solution of the following linear problem:

$$
\begin{cases}\frac{\partial v_{\varepsilon}^{\lambda}}{\partial t}+A\left(x, t, \frac{\partial}{\partial x}\right) v_{\varepsilon}^{\lambda}+\frac{\lambda}{\varepsilon} f^{\prime}(0) v_{\varepsilon}^{\lambda}=g, & (x, t) \in Q, \\ v_{\varepsilon}^{\lambda}(x, 0)=\psi(x), & x \in \bar{U}, \\ \pi_{0}^{\prime} v_{\varepsilon}^{\lambda}\left(x^{\prime}, t\right)=\phi\left(x^{\prime}, t\right), & \left(x^{\prime}, t\right) \in \Gamma .\end{cases}
$$


Proposition 2.4.2. - Assume $f(s)$ to be concave and the data to be nonnegative. Then $u_{\varepsilon}^{\lambda}(x, t) \geqslant v_{\varepsilon}^{\lambda}(x, t), \forall(x, t) \in \bar{Q}$, where $v_{\varepsilon}^{\lambda}$ is the solution of (2.4.1).

Proof. - The difference $w_{\varepsilon}^{\lambda}=u_{\varepsilon}^{\lambda}-v_{\varepsilon}^{\lambda}$ is a solution of the following problem:

$$
\begin{cases}\left(w_{\varepsilon}^{\lambda}\right)_{t}+A w_{\varepsilon}^{\lambda}+\lambda\left(f\left(\varepsilon^{-1} u_{\varepsilon}^{\lambda}\right)-f\left(\varepsilon^{-1}\left(u_{\varepsilon}^{\lambda}-w_{\varepsilon}^{\lambda}\right)\right)\right)=h_{\varepsilon}^{\tau}(x, t), & (x, t) \in Q, \\ w_{\varepsilon}^{\lambda}(x, 0)=0, & x \in U, \\ \pi_{0} w_{\varepsilon}^{\lambda}\left(x^{\prime}, t\right)=0, & \left(x^{\prime}, t\right) \in \Gamma,\end{cases}
$$

where the function

$$
h_{\varepsilon}=-f\left(\varepsilon^{-1} v_{\varepsilon}^{\lambda}\right)+\varepsilon^{-1} f^{\prime}(0) v_{\varepsilon}^{\lambda}
$$

s nonnegative since $f$ is concave. The maximum principle yields: $w_{\varepsilon}^{\lambda} \geqslant 0$ in $\bar{Q}$.

Propostrion 2.4.3. - If $u_{\varepsilon}^{\lambda} \geqslant 0$, then $u_{\varepsilon}^{\lambda}$ is a monotonically increasing function of $\varepsilon>0$.

Proof. - Let $w_{\varepsilon}^{\lambda}=\partial u_{\varepsilon}^{\lambda} / \partial \varepsilon$. Then $w_{\varepsilon}^{\lambda}$ is the solution of the problem

$$
\begin{cases}\frac{\partial w_{\varepsilon}^{\lambda}}{\partial t}+A w_{\varepsilon}^{\lambda}+\varepsilon^{-1} \lambda f^{\prime}\left(\varepsilon^{-1} u_{\varepsilon}^{\lambda}\right) w_{\varepsilon}^{\lambda}=\varepsilon^{-2} \lambda f^{\prime}\left(\varepsilon^{-1} u_{\varepsilon}^{\lambda}\right) u_{\varepsilon}^{\lambda}, & (x, t) \in Q, \\ w_{\varepsilon}^{\lambda}(x, 0)=0, & x \in \bar{U}, \\ \pi_{0} w_{\varepsilon}^{\lambda}\left(x^{\prime}, t\right)=0, & \left(x^{\prime}, t\right) \in \Gamma .\end{cases}
$$

As a consequence of the maximum principle, one finds $w_{\varepsilon}^{\lambda}(x, t) \geqslant 0, \forall(x, t) \in \bar{Q}$, since $f^{\prime}(s) \geqslant 0, u_{\varepsilon}^{\lambda} \geqslant 0$.

2.5. Special solutions of Cauchy's problem for the reduced operator.

In this section, we assume that

$$
\begin{aligned}
& A\left(x, t, \frac{\partial}{\partial x}\right)=-\Delta, \\
& U=\boldsymbol{R}^{n}, \\
& f_{0}(s)=\operatorname{sgn}(s), \quad \forall s \in \boldsymbol{R} \backslash\{0\}, f_{0}(0)=0, \\
& g(x, t)=0, \quad \forall(x, t) \in \boldsymbol{R}^{n} \times \boldsymbol{R}_{+} .
\end{aligned}
$$

We indicate the following two types of special nonnegative solutions of Cauchy's problem for the reduced operator. 
(i) Travelling waves' solutions:

$$
u^{\lambda}(x, t ; w, \xi)=\lambda w^{-1}(x \cdot \xi-w t)_{+}-\lambda|\xi|^{2} w^{-2}\left[1-\exp \left(-w|\xi|^{-2}(x \cdot \xi-w t)_{+}\right)\right]
$$

where $\xi \in \boldsymbol{R}^{n}, w \in \boldsymbol{R} \backslash\{0\}$ and $s_{+}=\max (s, 0)$.

(ii) Self-similar solutions.

These are solutions of the form

$$
u^{\lambda}(x, t)=t v\left(|x| t^{-1 / 2}\right)
$$

where $v(s), s \in \boldsymbol{R}_{+}$, are nonnegative solutions of the following ordinary differential equation

$$
-v^{\prime \prime}(s)-\left(\frac{s}{2}+\frac{n-1}{s}\right) v^{\prime}(s)+v(s)+\lambda \chi_{+}(v(s))=0, \quad s>0,
$$

so that $v(s) \geqslant 0$ is given by the formula

$$
v(s)=\left(s^{2}+2 n\right)\left[c_{1} \int_{1}^{s} \xi^{1-n}\left(\xi^{2}+2 n\right)^{-2} \exp \left(-\xi^{2} / 4\right) d \xi+c_{2}\right]-\lambda .
$$

If $n=1$, one finds the solution of the Cauchy problem

$$
\left\{\begin{array}{l}
\frac{\partial u^{\lambda}}{\partial t}-\frac{\partial^{2} u^{\lambda}}{\partial x^{2}}+\lambda \chi_{+}\left(u^{\lambda}\right)=0, \quad x \in \boldsymbol{R}, t>0 \\
u^{\lambda}(x, 0)=x_{+}^{2}
\end{array}\right.
$$

which has the form (2.5.2), where

$$
v(s)=\left\{\left(s^{2}+2\right)\left[a+b \int_{0}^{s}\left(\xi^{2}+2\right)^{-2} \exp \left(-\xi^{2} / 4\right) d \xi\right]-\lambda\right\} \mathscr{H}(s-\alpha) .
$$

In this case, the free boundary is a parabola $x=\alpha \sqrt{t}$. One gets a system of three equations for the parameters $a, b, \alpha$, which after elimination of $a$ and $b$ leads to the following functional equation for the free boundary parameter $\alpha$ :

$$
\left(\alpha^{2}+2\right)^{-1}-2 \alpha \exp \left(\alpha^{2} / 4\right) \int_{\alpha}^{\infty}\left(\xi^{2}+2\right)^{-2} \exp \left(-\xi^{2} / 4\right) d \xi=\lambda^{-1} .
$$

For $\forall \lambda>0$ the equation (2.5.7) has a well-defined solution $\alpha \in \boldsymbol{R}$. For $\lambda=2$, one gets the stationary solution $u(x, t)=x_{+}^{2}$. 


\subsection{Short time asymptotics.}

Consider the Cauchy problem:

$$
\left\{\begin{array}{l}
u_{t}^{\lambda}-u_{x x}^{\lambda}+\lambda \chi_{+}\left(u^{\lambda}\right)=0, \quad x \in \boldsymbol{R}, t>0 \\
u^{\lambda}(x, 0)=\psi(x)
\end{array}\right.
$$

where $\psi(x) \equiv 0$ for $x<0, \psi(x)=\gamma x_{+}^{2}+O\left(x_{+}^{3}\right)$ for $x_{+} \rightarrow 0$ and $\psi(x) \leqslant C x^{2}, \forall x \in \boldsymbol{R}_{+}$ with some constant $C>0$.

Denote by $w^{\lambda}(x, t)$ the corresponding self-similar solution:

$$
\left\{\begin{array}{l}
w_{t}^{\lambda}-w_{x s}^{\lambda}+\lambda \chi_{+}\left(w^{\lambda}\right)=0, \quad x \in \boldsymbol{R}, t>0, \\
w^{\lambda}(x, 0)=\gamma x_{+}^{2}
\end{array}\right.
$$

$w^{\lambda}(x, t)$ being defined by the formula $w^{\lambda}(x, t)=t v^{\lambda}(x / \sqrt{t})$ with $v^{\lambda}(s)$ given by (2.5.6), (2.5.7).

Then the following estimate holds:

$$
\sup _{(x, t) \in Q_{a, T}^{\delta}}\left|u^{\lambda}(x, t)-w^{\lambda}(x, t)\right| \leqslant C_{a, T} \delta^{3}, \quad \forall \delta>0
$$

where $Q_{a, T}^{\delta}=\left\{(x, t): 0 \leqslant t \leqslant T \delta^{2},|x| \leqslant a \delta\right\}$ and the constant $C_{a, T}$ does not depend of $\delta$.

One proves (2.6.3) using the method introduced in [21].

\subsection{Asymptotios for $\lambda \rightarrow \infty$.}

In order to avoid unnecessary technical complications we consider here the case of one space variable $x \in U=(-1,1)$ and of special (constant) initial and boundary conditions. Namely, consider the problem $\mathfrak{A}_{\varepsilon}^{\lambda}$ :

$$
\begin{cases}u_{t}^{\lambda}-u_{x x}^{\lambda}+\lambda \chi_{+}\left(u^{\lambda}\right)=0, & (x, t) \in U \times \boldsymbol{R}_{+}, \\ u^{\lambda}(x, 0)=1, & x \in U, \\ \pi_{0} u^{\lambda}\left(x^{\prime}, t\right)=1, & \left(x^{\prime}, t\right) \in \partial U \times \boldsymbol{R}_{+} .\end{cases}
$$

Let $v(x, t)$ be the solution of the problem:

$$
\begin{cases}v_{t}-v_{x x}=1, & (x, t) \in U \times \boldsymbol{R}_{+}, \\ v(x, 0)=0, & x \in U, \\ \pi_{0} v\left(x^{\prime}, t\right)=0, & \left(x^{\prime}, t\right) \in \partial U \times \boldsymbol{R}_{+},\end{cases}
$$

and let $t_{c}(\lambda)$ be defined by the relation:

$$
v\left(0, t_{c}(\lambda)\right)=\lambda^{-1} .
$$


Then for $t \in\left[0, t_{e}(\lambda)\right)$ holds:

$$
u^{\lambda}(x, t)=1-\lambda v(x, t)
$$

Denote

$$
\gamma=\gamma(\lambda)=\lambda\left(1-v\left(0, t_{c}(\lambda)\right)\right)
$$

and let $w_{\gamma}^{\lambda}(x, t)$ be the self-similar solution of the Cauchy problem:

$$
\left\{\begin{array}{l}
\left(w_{\gamma}^{\lambda}\right)_{t}-\left(w_{\gamma}^{\lambda}\right)_{x x}+\lambda \chi_{+}\left(w_{\gamma}^{\lambda}\right)=0, \quad(x, t) \in \boldsymbol{R} \times \boldsymbol{R}_{+}, \\
w_{\gamma}^{\lambda}(x, 0)=\gamma x^{2} .
\end{array}\right.
$$

Thzoreim 2.7.1. - For any constants $T>0, a>0$, there exists a constant $C=O(T, a)$ such that for the solution $u^{\lambda}(x, t)$ the following inequality holds:

$$
\sup _{\substack{0 \leqslant t-t_{t}(\lambda) \leqslant T \lambda^{-1} \\|x| \leqslant a \lambda^{-1 / 2}}}\left|u^{\lambda}(x, t)-w_{\gamma}^{\lambda}\left(x, t-t_{0}(\lambda)\right)\right| \leqslant C(T, a) \lambda^{-3 / 2}
$$

where $t_{c}(\lambda), \gamma, w_{\gamma}^{\lambda}(x, t)$ are defined by (2.7.2)-(2.7.5).

REMARK 2.7.2. - One finds easily that $t_{c}(\lambda)=\lambda^{-1}+o\left(\lambda^{-1}\right)$. Furthermore, for $0 \leqslant t<t_{c}(\lambda)$ the following asymptotic formula holds:

$$
u^{\lambda}(x, t)=(1-\lambda t)+O\left(\lambda^{-1}\right) \quad \text { for } \lambda \rightarrow \infty
$$

while for $\sqrt{\bar{\lambda}} t \gg 1$ one has:

$$
u^{\lambda}(x, t) \sim u_{\infty}^{\lambda}(x), \quad \lambda \rightarrow \infty
$$

where

$$
u_{\infty}^{\lambda}(x)=(\lambda / 2)\left(|x|-1+\sqrt{2 \lambda^{-1}}\right)_{+}^{2}
$$

is the solution of the corresponding stationary problem.

For $t_{c}(\lambda)<t<o \lambda^{-1 / 2}$ one has:

$$
u^{\lambda}(x, t) \sim v\left(\lambda\left(t-t_{e}(\lambda)\right), \sqrt{\lambda} \operatorname{dist}(x, \partial U)\right), \quad \lambda \rightarrow \infty,
$$

where $v(\tau, \zeta)$ is the solution of the problem:

$$
\begin{cases}v_{\tau}-v_{\zeta \zeta}+\chi_{+}(v)=0, & (\zeta, \tau) \in \boldsymbol{R}_{+} \times \boldsymbol{R}_{+} \\ v(\zeta, 0)=1, & \zeta \in \boldsymbol{R}_{+} \\ v(0, \tau)=1, & \tau \in \boldsymbol{R}_{+}\end{cases}
$$


Introducing $w=v_{\tau}$ one can reformulate (2.7.10) as the following slightly modified Stefan problem:

$$
\begin{cases}w_{\tau}-w_{\zeta \zeta}=0, & 0<\zeta<s(\tau), \tau \in \boldsymbol{R}_{+}, \\ w(0, \tau)=0, & \tau \in \boldsymbol{R}_{+}, \\ w(\zeta, 0)=-1, & \zeta \in \boldsymbol{R}_{+}, \\ w(s(\tau), \tau)=\mathbf{0}, w_{x}(s(\tau), \tau)+\dot{s}(\tau)=0, & \tau \in \boldsymbol{R}_{+}, \\ s(\infty)=\sqrt{2}, & \end{cases}
$$

the eurve $\zeta=s(\tau)$ being the free boundary for the solution $w(\zeta, \tau)$ of $(2.7 .11)$.

One has also:

$$
\lim _{\tau \rightarrow \infty} v(\zeta, \tau)=(1-\zeta / \sqrt{2})_{+}^{2}
$$

The proof of Theorem 2.7.1 and the claims stated in Remark 2.7.2, as well as the corresponding generalizations, will be presented elsewhere.

$$
* * *
$$

This research has been partially supported by the European Research Office of the U.S. Army under the contract No. DAJA-37-82-C-0731.

\section{REFERENCES}

[1] C. BAIOCCHI, Su un problema a frontiera libera connesso a questioni di idraulica, Ann. Mat. Pura ed Appl. (4), 92 (1972), pp. 107-127.

[2] C. M. BRAUneR - B. NicolaEnKo, Singular perturbations and free boundary problems, in: Computing Methods in Applied Soiences and Engineering; R. Glowinsky and J.-L. Lions, ed., North-Holland, 1980.

[3] H. BRÉzis, Opérateurs maximaux monotones, North-Holland, 1973.

[4] H. BREzTs - D. KINDERLEHRER, The smoothness of solutions to nonlinear variational inequalities, Indiana Math. Journ., 23 (1974), pp. 831-844.

[5] I. EKELAND - R. Témam, Oonvex analysis and variational problems, North-Holland, 1976.

[6] L. S. FRANK - E. W. C. VAN GROESEN, Singular perturbations of an ellipic operator with discontinuous nonlinearity, in: Analytical and Numerical Approaches to Asymptotic Problems in Analysis, O. Axelsson, L. S. Frank, A. van der Sluis (eds.), North-Holland, 1981.

[7] L. S. FRANK - W. D. WENDT, On a singular perturbation in the kinetic theory of enzymes, in: Theory and applications of singular perturbations, W. Eckhaus, E. M. de Jager (eds.), Springer, 1982.

[8] L. S. FRANK - W. D. WENDT, Sur une perturbation singutière en théorie des enzymes, C.R.A.S. Paris, t. 294 (1982), pp. 741-744; Solutions asymptotiques pour une classe de 
perturbations singulières elliptiques semilinéaires, C.R.A.S. Paris, t. 295 (1982), pp. 451-454; Sur une perturbation singulière parabolique en théorie cinétique des enzymes, C.R.A.S. Paris, t. 295 (1982), pp. 731-734.

[9] L. S. FRANK - W. D. WENDT, On an elliptic operator with discontinuous nonlinearity, J. Diff. Eq., 54, No. 1 (1984), pp. 1-18.

[10] Free boundary problems I, II, Proceedings of a Seminar at Pavia, E. Magenes (ed.), Rome, 1980.

[11] A. Friedman, Variational principles and free boundary problems, Pure and Applied Mathematics, Wiley Interscience Series, 1982.

[12] R. Goldman - O. Kedmi - E. Katchalski, Papain-Colloidin Membranes II, Biochemistry, 10 (1971), pp. 165-172.

[13] D. KInDERLEHreR - L. NirenberG, The smoothness of the free boundary in the one phase Stefan problem, Séminaire Collège de France, 1976.

[14] H. LEWY - G. STAMPACCHIA, On the regularity of the solution of a variational inequality, Comm. Pure Appl. Math., 22 (1969), pp. 153-188.

[15] J.-L. Lions - G. Stampacchia, Variational inequalities, Comm. Pure Appl. Math., 20 (1967), pp. 493-519.

[16] J.-L. LIons, Perturbations singutières dans les problèmes aux limites et en contrôle optimale, Springer, 1973.

[17] R. TÉmam, Remarks on a free boundary problem arising in plasma physics, Comm. PDE, 2 (1977), pp. 563-585.

[18] A. TRENOGIN, Development and applications of Vishik-Lyusternik's asymptotic method, Uspekhi Math. Nauk., 25 (1970), pp. 123-156.

[19] M. VISHIK - L. LXUSTERNIK, Regular degeneration and boundary layer for linear differential equations with small parameter, Uspekhi Mat. Nauk, 12 (1957), pp. 3-122, AMS Transl., (2), 20 (1962), pp. 239-364.

[20] M. VISHIK - L. LYUSTERNIK, The asymptotic behaviour of solutions of linear aifferential equations with large or quickly varying coefficients and conditions at the boundary, Uspekhi Mat. Nauk, 15 (1960), pp. 27-95.

[21] S. KaMrN, The asymptotic behaviour of the solution of the filtration equation, Israel $\mathrm{J}$. Math., 14 (1973), pp. 76-87. 